\title{
Effect of an external electric field on the dissociation energy and the electron density properties: The case of the hydrogen bonded dimer HF...HF
}

\author{
Ignasi Mata, ${ }^{1, a)}$ Elies Molins, ${ }^{1}$ Ibon Alkorta, ${ }^{2}$ and Enrique Espinosa ${ }^{3}$ \\ ${ }_{1}^{1}$ Institut de Ciència de Materials de Barcelona (ICMAB-CSIC), Campus UAB, 08193 Bellaterra, Spain \\ ${ }^{2}$ Instituto de Química Médica (IQM-CSIC), Juan de la Cierva, 3, 28006 Madrid, Spain \\ ${ }^{3}$ Laboratoire de Cristallographie et Modélisation des Matériaux Minéraux et Biologiques $\left(\mathrm{LCM}^{3} \mathrm{~B}\right)$, \\ UMR CNRS 7036, Institut Jean Barriol, Nancy-Université, BP 239, 54506 Vandoeuvre-lès-Nancy, France
}

(Received 18 July 2008; accepted 15 December 2008; published online 28 January 2009)

\begin{abstract}
The effect of a homogeneous external electric field parallel to the hydrogen bond in the $\mathrm{FH} \cdots \mathrm{FH}$ dimer has been studied by theoretical methods. The quantum theory of atoms in molecules methodology has been used for analyzing the electron distribution of the dimer, calculated with different hydrogen bond distances and external field magnitudes. It is shown that an electric field in the opposite direction to the dipole moment of the system strengthens the interaction due to a larger mutual polarization between both molecules and increases the covalent character of the hydrogen bond, while an external field in the opposite direction has the inverse effect. The properties of the complex at its equilibrium geometry with applied field have been calculated, showing that dependencies between hydrogen bond distance, dissociation energy, and properties derived from the topological analysis of the electron distribution are analogous to those observed in families of $\mathrm{XDH} \cdots \mathrm{AY}$ complexes. The application of an external field appears as a useful tool for studying the effect of the atomic environment on the hydrogen bond interaction. In the case of $\mathrm{FH} \cdots \mathrm{FH}$, both the kinetic energy density and the curvature of the electron density along the hydrogen bond at the bond critical point present a surprisingly good linear dependence on the dissociation energy. The interaction energy can be modeled by the sum of two exponential terms that depend on both the hydrogen bond distance and the applied electric field. Moreover, as indicated by the resulting interaction energy observed upon application of different external fields, the equilibrium distance varies linearly with the external field, and the dependence of the dissociation energy on either the hydrogen bond distance or the external electric field is demonstrated to be exponential. (C) 2009 American Institute of Physics. [DOI: 10.1063/1.3065972]
\end{abstract}

\section{INTRODUCTION}

Quantum theory of atoms in molecules (QTAIM) ${ }^{1,2}$ allows the characterization of interatomic interactions and the calculation of some atomic properties directly from the electron density distribution. For this reason, the QTAIM methodology has been extensively used in the analysis of experimental electron distributions in crystals. Although many kinds of bonding interactions observed in crystals have been analyzed in terms of QTAIM, hydrogen bonding has deserved special attention due to its interest in many fields of research, from biochemistry to crystal engineering and pharmaceutics. ${ }^{3}$

The application of QTAIM to hydrogen bonds has shown the existence of simple dependencies between the properties of the electron distribution in the bonding region and the energy of the interaction. ${ }^{4,5}$ Moreover, similar dependencies between these properties and the bonding distance have been observed from the analysis of experimental elec-

\footnotetext{
*This work is dedicated to the memory of Niels K. Hansen (1950-2007), Professor of Crystallography and Chemical Physics at $\mathrm{LCM}^{3} \mathrm{~B}$ (University of Nancy).

a)Electronic mail: imata@icmab.es.
}

tron distributions in crystals. ${ }^{6}$ This is related to the existence of close relationships between the hydrogen bond distance and the interaction energy. ${ }^{7}$ Those results have been used for deriving an empirical interaction potential for the hydrogen bond from experimental data of electron distributions in crystals. $^{8}$

The relationships between interaction energy, bonding distance, and properties of the electron distribution are frequently observed in theoretical studies of families of similar hydrogen bonded complexes. ${ }^{4,5}$ While in these studies the complexes are supposed to be isolated, in most cases of interest the interaction takes place in an environment that is susceptible to perturbing the interaction, as a polar solvent or a crystal. It has been shown that the environment of the interaction can be described in terms of an isotropic external field parallel to the hydrogen bond, ${ }^{9}$ this approach being successfully used in theoretical studies dealing with environment effects on IR (Ref. 10) and NMR spectra. ${ }^{11}$ External electric fields have been also applied to the analysis of the electron distribution in the framework of QTAIM, for example, in the modelization of proton transfer induced by the solvent in acid-base complexes ${ }^{12}$ or for representing the crystal environment in dihydrogen bonded systems. ${ }^{13}$ 
In previous works covering both unperturbed ${ }^{14}$ and perturbed ${ }^{15}$ systems, the relationship between the molecular orbitals (MOs) involved in both the hydrogen bond and the electron distribution in the covalent bond region has been shown. Here, external fields are used in order to study the effect of a perturbation, representing the atomic environment, on the hydrogen bond interaction. The influence of the electric field on the MOs and the electron distribution is analyzed in detail, showing the polarization of the orbitals and the effect of this field on the strength of the interaction. In this work, while calculations are performed in gas phase, the results are taken as a good approximation to the environment effect on hydrogen bonds in condensed matter. The modulus of the applied electric field was chosen to range from 0.01 to 0.05 a.u. (0.01 a.u. $=5.1422082 \times 10^{9} \mathrm{~V} / \mathrm{m}$ $\left.=1.7152447 \times 10^{5} \mathrm{esu}\right)$. The lowest values in this range typically fall within those felt by a molecule in a crystal (roughly $3-15 \mathrm{GV} / \mathrm{m})^{16,17}$ and in proteins and enzymes (roughly $5 \mathrm{GV} / \mathrm{m}){ }^{18,19}$

The simple system chosen for this analysis was the linear $\mathrm{FH} \cdots \mathrm{FH}$ dimer with a cylindrical symmetry imposed. The comparison with a detailed study of the most stable nonlinear $(\mathrm{FH})_{2}$ at its bent geometry with no electric field applied $^{20}$ shows that the crude symmetrical approximation, which eases the following discussion, has no qualitative effect on the electron distribution, in particular in the intermolecular region. As the polarization of the MOs induced by an external field parallel to the hydrogen bond is expected to be similar to the effect of the environment on the intermolecular interaction, ${ }^{9}$ electric fields were applied along the symmetry axis only. Hereafter, the left and right molecules in the $\mathrm{FH} \cdots \mathrm{FH}$ interaction are taken as the donor and the acceptor ones, respectively.

\section{COMPUTATIONAL DETAILS}

Calculations were performed with GAUSSIAN $-03^{21}$ at the MP2/6-311++G( $d, p)$ computational level $\mathrm{l}^{22,23}$ on a $\mathrm{F}-\mathrm{H} \cdots \mathrm{F}-\mathrm{H}$ complex and on a $\mathrm{F}-\mathrm{H}$ molecule, both of them with a $C_{\infty v v}$ symmetry imposed. In the linear dimer, the $\mathrm{H} \cdots \mathrm{F}$ distance $\left(d_{\mathrm{FH}}\right)$ was varied in steps of $0.1 \AA$ along the range of 1.2-3.0 $\AA$. For each $d_{\mathrm{FH}}$, electric fields parallel to the symmetry axis $(\mathbf{E}=\boldsymbol{\epsilon \mathbf { Z }})$ were applied in steps of 0.01 a.u. from $\epsilon=-0.05$ to $\epsilon=0.05$ a.u., taking as positive the direction from the hydrogen donor to the fluorine acceptor in the $\mathrm{H} \cdots \mathrm{F}$ interaction. For each $\left(d_{\mathrm{FH}}, \epsilon\right)$ pair, both $\mathrm{F}-\mathrm{H}$ distances were relaxed. Besides this calculation, the geometries of the dimer and the monomer were relaxed upon application of the same external fields (always keeping the $C_{\infty o v}$ symmetry).

In order to further check the adequacy of the linear dimer approximation, the geometry of the $(\mathrm{FH})_{2}$ complex upon $\epsilon$ was calculated keeping the donor molecule and the acceptor fluorine aligned, while varying the angle between the acceptor molecule and the hydrogen bond along with $d_{\mathrm{FH}}$ and both FH distances. According to these calculations, the external field has a strong effect on the geometry of the $(\mathrm{FH})_{2}$ dimer, presenting a bent conformation for $-0.01 \leq \epsilon$ $\leq 0.01$ a.u. only. With $\epsilon \geq 0.02$ a.u. the complex deviates by less than $1^{\circ}$ from linearity, whereas with $\epsilon \leq-0.02$ a.u. the

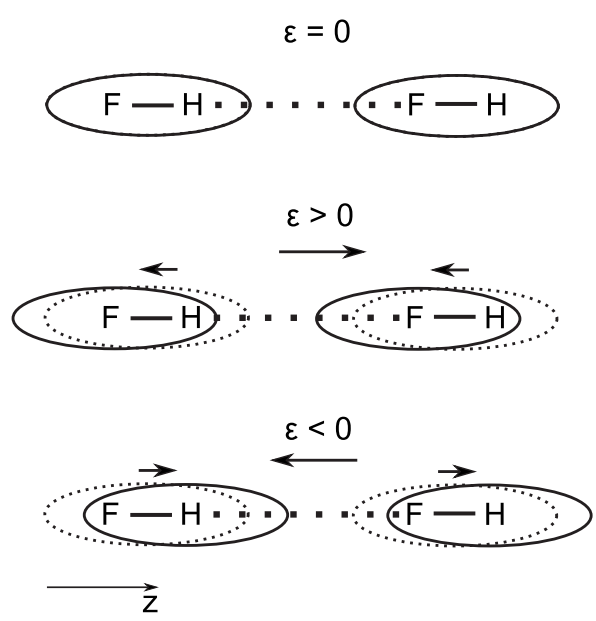

FIG. 1. Polarization of the electron density in the MOs of the linear $(\mathrm{FH})_{2}$ dimer induced by the external field.

complex collapses to FH $\cdots \mathrm{HF}$. As the interest here does not concern the effect of an external electric field on the $(\mathrm{FH})_{2}$ complex itself, but on the effect of this field on the hydrogen bond interaction, the linear conformation of $(\mathrm{FH})_{2}$, which can be assumed for most of the positive values of $\epsilon$, was used in order to extend the range of applied fields to negative $\epsilon$.

The electron density topology of the systems was analyzed with the AIMPAC package,${ }^{24}$ while the integration of the atomic properties within the atomic basins was performed with AIMPAC and MORPHY98. ${ }^{25}$ The quality of the atomic integration was checked with the value of the integrated Laplacian, which must be zero within each atomic basin. In all cases, the integrated Laplacian remained, in absolute value, smaller than 0.001 a.u., ensuring small errors in energies and charges. ${ }^{26}$

As the electron density is polarized in the direction opposite to the electric field (Fig. 1), the positive field displaces the electron clouds in the acceptor and in the donor molecules toward and away from the hydrogen bonding region, respectively, while the negative field has the opposite effect. As shown in the following discussion, most of the considered properties vary monotonically within the range of applied fields. In order to simplify the following discussion, both positive and negative fields will be treated together. Hereafter, the increase or the decrease in the field does not refer to the field module but to the external field along the bonding axis, which depends on the sign convention adopted. Thus, an increase in the field is interpreted as an increase in $|\mathbf{E}|$ when $\epsilon>0$ or as a decrease in $|\mathbf{E}|$ if $\epsilon<0$.

\section{RESULTS AND DISCUSSION}

\section{A. The linear dimer without external electric field}

According to the QTAIM theory, zero-flux surfaces $S$ are defined by the condition $\nabla \rho(\mathbf{r}) \cdot \mathbf{n}(\mathbf{r})=0, \forall \mathbf{r} \in S, \mathbf{n}$ being the unit vector perpendicular to $S$ at $\mathbf{r}$. The zero-flux surfaces define the boundaries of atoms, allowing a partition of the space in atomic volumes $\Omega$ (atomic basins). Hence, the calculation of atomic and molecular properties $\left(P_{\Omega}\right.$ and $P$ $=\Sigma_{\Omega} P_{\Omega}$, respectively), such as the charge and the energy, can be done by integration on these basins. In addition, any 
bonding interaction between two atoms is exhibited in the electron density distribution $[\rho(\mathbf{r})]$ as a topological saddle conformation around the interatomic zero-flux surface $(S)$. At $S$, bond critical points (BCPs) appear, where the gradient of $\rho(\mathbf{r})$ vanishes $[\nabla \rho(\mathbf{r})=0]$, and the electron density is a minimum in the direction parallel to the bond and a maximum in the perpendicular plane. Two gradient lines starting at the corresponding nuclei and ending at $\mathrm{BCP}$ form the bond path, which is considered as an identifier of the interaction. ${ }^{27}$

Several properties of the electron distribution at BCP are used for the characterization of the interaction. These critical point properties are the value of $\rho(\mathbf{r})$ at $\operatorname{BCP}\left(\rho_{b}\right)$, as well as the three eigenvalues and the trace of the Hessian matrix of $\rho(\mathbf{r})$ at the same point. The three eigenvalues correspond to the curvatures of $\rho(\mathbf{r})$ : one parallel to the bond path direction $\left(\lambda_{\|, b}\right)$, which is positive, and two perpendicular to that direction $\left(\lambda_{\perp, b}\right.$, both equal in the linear $(\mathrm{FH})_{2}$ due to the cylindrical symmetry of this complex), which are negative. The trace of the Hessian matrix is the Laplacian of $\rho(\mathbf{r})\left(\nabla^{2} \rho_{b}\right)$, which is equal to the sum of the three curvatures. Other properties of interest that are also derived from the wave function are the electron kinetic and potential energy densities at BCP $\left(G_{b}>0\right.$ and $V_{b}<0$, respectively), which are related to the Laplacian by the local form of the virial theorem (in a.u.), ${ }^{1}$

$$
\frac{1}{4} \nabla^{2} \rho_{b}=2 G_{b}+V_{b}
$$

as well as the total energy density $\left(H_{b}=G_{b}+V_{b}\right)$. While the energy densities cannot be straightforwardly derived from experimental data, they can be estimated from experimentally determined $\rho(\mathbf{r})$ distributions for closed shell interactions. $^{7,28-30}$

It has been shown from the analysis of these topological and energetic properties that the nature of the $\mathrm{F} \cdot \mathrm{H}$ interaction depends on the interatomic distance $d_{\mathrm{FH}} \cdot{ }^{14}$ For large enough $d_{\mathrm{FH}}$ distances, $\nabla^{2} \rho_{b}>0, H_{b}>0$, and $\left|V_{b}\right| / G_{b}<1$, and the interaction corresponds to a pure closed shell type (zone I). For medium-range $d_{\mathrm{FH}}$ distances, where $\nabla^{2} \rho_{b}>0, H_{b}$ $<0$ and $2<\left|V_{b}\right| / G_{b}<1$, these electronic properties are associated to a closed-shell interaction with a significant covalent character (zone II). According to the natural bond orbital (NBO) analysis, ${ }^{31}$ it is in zone II that the reorganization of the MOs giving rise to the bonding orbital between $\mathrm{F}$ and $\mathrm{H}$ takes place. For shorter $d_{\mathrm{FH}}$ distances, $\nabla^{2} \rho_{b}<0, H_{b}<0$, and $\left|V_{b}\right| / G_{b}>2$, and the interaction is purely covalent (zone III). Hydrogen bonds of weak and medium strengths fall in zone I, while strong hydrogen bonds are observed in zone II, and very strong ones (in most cases symmetric) in zone III. ${ }^{14,32}$

According to this classification, the range of $d_{\mathrm{FH}}$ distances considered here spans zones I and II, being the border between these zones at $d_{\mathrm{FH}} \sim 1.5 \AA$, as observed from $H_{b}$ $=0$ or $\left|V_{b}\right| / G_{b}=1$. In all the interaction ranges, the absolute values of all the topological and energetic properties but $H_{b}$ and $\nabla^{2} \rho_{b}$ grow exponentially as $d_{\mathrm{FH}}$ is reduced. While the increase in $\left|V_{b}\right|$ is interpreted as a stronger pressure exerted by the system on the electron density in the hydrogen bonding region, which concentrates the electron distribution along the bond path and increases $\rho_{b}$, the increase in $G_{b}$ is interpreted as the pressure exerted by these electrons against the atomic basins as the closed shells are brought together. ${ }^{7} G_{b}$

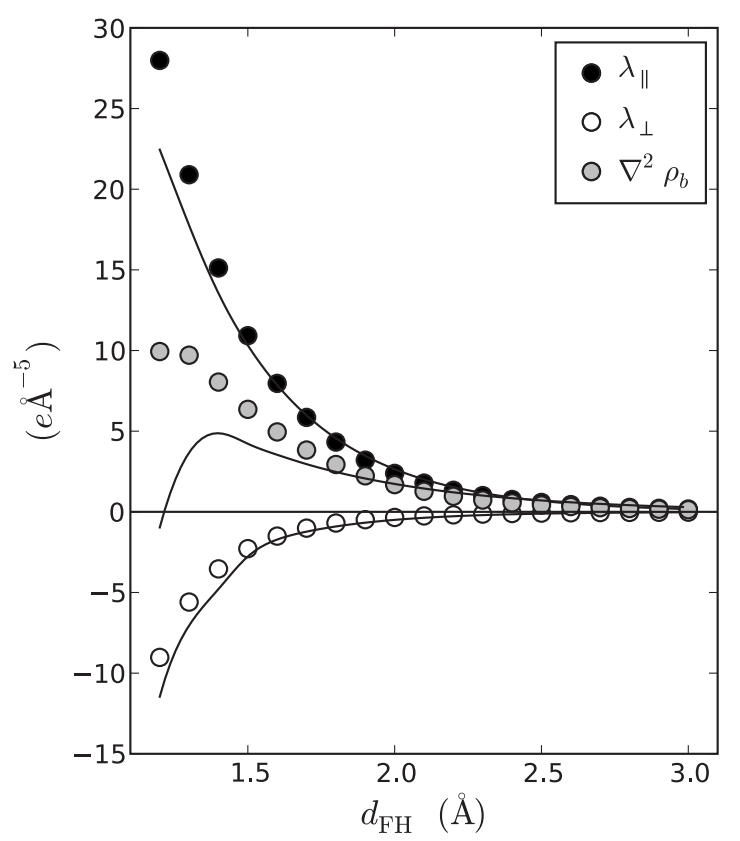

FIG. 2. $\lambda_{\|, b}, \lambda_{\perp, b}$, and $\nabla^{2} \rho_{b}$ vs $d_{\mathrm{FH}}$ for the linear $(\mathrm{FH})_{2}$ with $\epsilon=0$. Solid lines are the joint functions fitted to a set of FH $\cdots$ FX complexes in Ref. 14.

and $V_{b}$ are mostly influenced by the Pauli repulsion and the stabilizing effect of the electric field, respectively. Indeed, the electrostatic part of the pressure over the electrons in the bonding region comes from the electric field, which pushes them toward the bond axis. ${ }^{33}$ Thus, the increase in $\left|V_{b}\right|$ is accompanied by an increase in $\left|\lambda_{\perp, b}\right|$. Moreover, as $d_{\mathrm{FH}}$ shortens, the Pauli repulsion between the closed shells becomes stronger, increasing the depletion of $\rho(\mathbf{r})$ by the increase in the electron kinetic energy $G_{b}$, leading to a $\rho(\mathbf{r})$ steeper along the bond path and the concomitant increase in $\lambda_{\|, b}{ }^{29}$

$H_{b}$ and $\nabla^{2} \rho_{b}$ are related to the balance between both the stabilizing action of the electric field in the bonding region and the Pauli repulsion between the closed shells. As the molecules approach, both $H_{b}$ and $\nabla^{2} \rho_{b}$ increase until a maximum is reached, then they drop to very negative values as the reorganization of the MOs relaxes the Pauli repulsion and the transition to a covalent bond takes place. While $H_{b}$ exhibits its maximum in zone I and is negative in zone II, $\nabla^{2} \rho_{b}$ is only negative in zone III and is positive in all the interaction range considered in this work.

In the linear $(\mathrm{HF})_{2}$, the dependencies of the electron properties at the hydrogen bond $\mathrm{BCP}$ on $d_{\mathrm{FH}}$ are similar to those reported for (i) the $(\mathrm{HF})_{2}$ complex at the bent configuration, with its geometry relaxed except for the hydrogen bonding $d_{\mathrm{FH}}$ distance (hereafter optimized dimer), ${ }^{20}$ and for (ii) a large set of $\mathrm{XH} \cdots \mathrm{FY}$ complexes at optimized geometries. ${ }^{14}$ The only significant differences with the functions fitted to these properties in (ii) are a smaller $\rho_{b}$ and larger $\lambda_{\|, b}, G_{b}, \nabla^{2} \rho_{b}$, and $H_{b}$ for $d_{\mathrm{FH}} \lesssim 2 \AA$, together with a shift in the maxima of $\nabla^{2} \rho_{b}$ and $H_{b}$ to shorter $d_{\mathrm{FH}}$ in the linear dimer (Fig. 2). Moreover, (i) shows both maxima at larger $d_{\mathrm{FH}}\left(1.4 \AA\right.$ for $\nabla^{2} \rho_{b}$ and $2.1 \AA$ for $H_{b}$ in the optimized dimer and $1.2 \AA$ for $\nabla^{2} \rho_{b}$ and $1.7 \AA$ for $H_{b}$ in the linear 


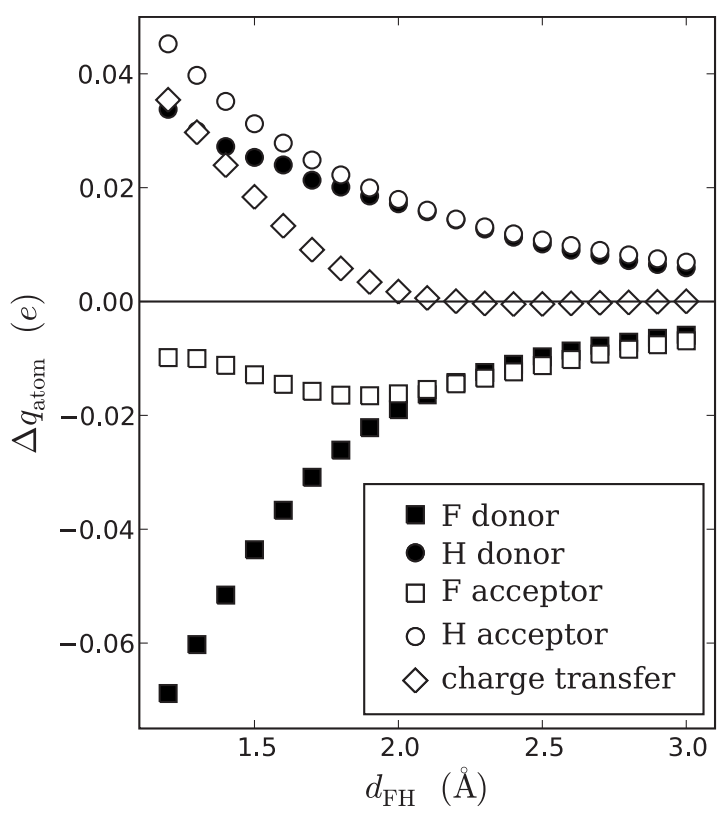

(a)

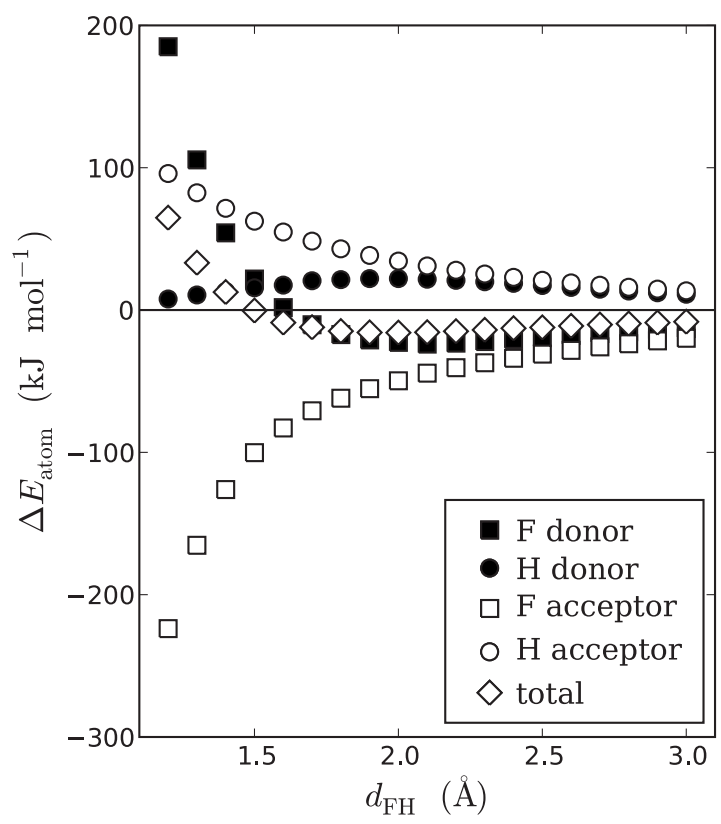

(b)

FIG. 3. Variation in the integrated (a) charges $\left(\Delta q_{\text {atom }}\right)$ and (b) energies $\left(\Delta E_{\text {atom }}\right)$ for the four atoms in the complex with respect to the values calculated for the isolated monomers $(\Delta=$ complex-monomers).

dimer). All these differences are explained by the reorganization of the MOs starting at shorter distances in the linear $(\mathrm{FH})_{2}$ dimer.

At $\epsilon=0$, the dependencies of the integrated properties (charge and energy) on $d_{\mathrm{FH}}$ show qualitatively different behaviors for short and long distances, being the transition situated at $d_{\mathrm{FH}} \approx 2.0 \AA$ (Fig. 3). For $d_{\mathrm{FH}} \gtrsim 2.0 \AA$, intermolecular charge transfer is not significant but increases as the molecules approach, giving rise to a larger charge separation within the FH entities. Moreover, the molecules are stabilized due to the decrease in the energy of the F-atoms, which is only partly compensated by the destabilization of the
$\mathrm{H}$-atoms. As $d_{\mathrm{FH}}$ is reduced, additional intramolecular charge transfer in each molecule is explained by the polarization of the MOs, which is induced by the electric field from the other one. This mutual polarization results in a stabilization of the complex.

For $d_{\mathrm{FH}} \lesssim 2.0 \AA$, electron transfer from the acceptor to the donor molecule takes place and is more important as the molecules approach. The energies of the central atoms in the complex decrease, while those of the terminal atoms increase. The overall energy of the complex is dominated by the terminal atoms, as observed in the $(\mathrm{FH})_{2}$ dimer with optimized geometry. ${ }^{20}$ This can be explained by the reorganization of the MOs involved in the transition from the hydrogen bond to the covalent bond, which implies the breaking of the covalent bond in both molecules and the concomitant destabilization of the terminal atoms in the complex.

The weakening of both covalent bonds below $d_{\mathrm{FH}}$ $\approx 2.0 \AA$ can be observed on the increase in their bonding distances and on the critical point properties at the corresponding BCPs. Thus, while these properties remain constant above $d_{\mathrm{FH}} \approx 2.0 \AA$, below this distance $\rho_{b}$ and $\left|V_{b}\right|$ decrease and $G_{b}$ increases, with the concomitant decrease in $\left|\nabla^{2} \rho_{b}\right|$ and $\left|H_{b}\right|$ (Fig. 4), both negative as expected for a covalent interaction.

As far as the integrated properties are concerned, the only qualitative difference between the linear and the optimized $(\mathrm{FH})_{2}$ dimers $^{20}$ appears on the hydrogen charge of the donor, which decreases monotonically with $d_{\mathrm{FH}}$ on the former while on the latter presents a maximum close to the equilibrium position $\left(d_{\mathrm{FH}}=1.831 \AA\right)$ because, in this last case, the charge transfer at short distances balances the electronic depopulation of this atom due to the hydrogen bond formation. ${ }^{34}$ Moreover, charge transfer on the linear dimer starts at shorter $d_{\mathrm{FH}}$ and is smaller than in the optimized dimer. Thus, whereas charge transfer on the optimized dimer starts at $d_{\mathrm{FH}} \approx 2.5 \AA$ and equals $0.04 e$ at $d_{\mathrm{FH}}=1.5 \AA$, on the linear dimer it starts at $d_{\mathrm{FH}} \approx 2.0 \AA$ and is only of $0.018 e$ at $d_{\mathrm{FH}}=1.5 \AA$.

\section{$B$. The effect of the external electric field on the topological and energetic properties at BCP}

The external electric field $(\epsilon)$ polarizes the orbitals of both molecules, displacing their electron clouds (Fig. 1) and therefore the hydrogen bond BCP in the direction opposite to the field. The shift in the BCP position is proportional to $\epsilon$ and $d_{\mathrm{FH}}$, and the distance from the acceptor fluorine to BCP $\left(d_{\mathrm{Fb}}\right)$ can be expressed in terms of a bilinear function on these variables whose coefficients can be determined by a linear squares fitting $[R=0.9996$ for 209 points, Fig. 5(a)],

$$
d_{\mathrm{F} b}=0.91(3) \epsilon d_{\mathrm{FH}}+0.504(1) d_{\mathrm{FH}}-0.91(7) \epsilon+0.259(2) \text {. }
$$

For the range of $d_{\mathrm{FH}}$ 's considered here, the first term in Eq. (2) is, in absolute value, larger than the third one, agreeing with the polarization of the closed shells represented in Fig. 1. The terms remaining in Eq. (2) when $\epsilon=0$ correspond to a linear dependence with slope $\approx 0.5$, pointing that the $\mathrm{H}$ and $\mathrm{F}$ atomic basins of the donor and the acceptor molecules, 


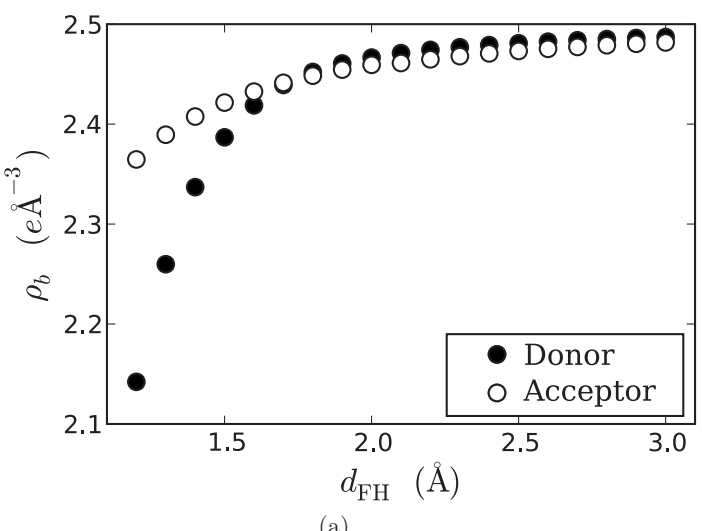

(a)

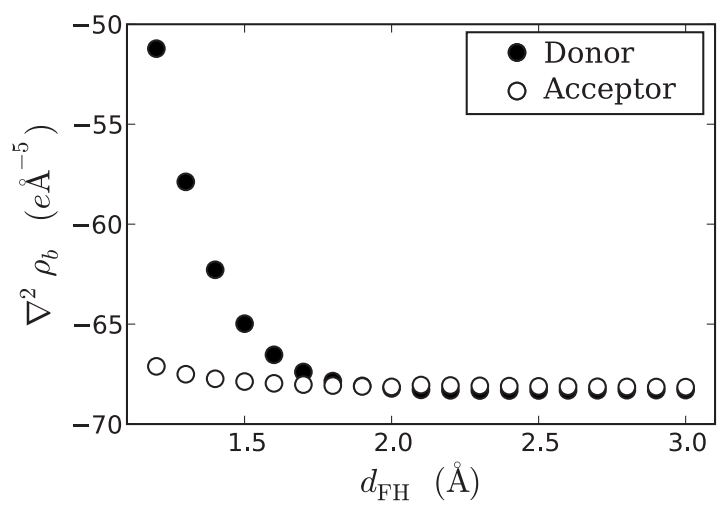

(b)

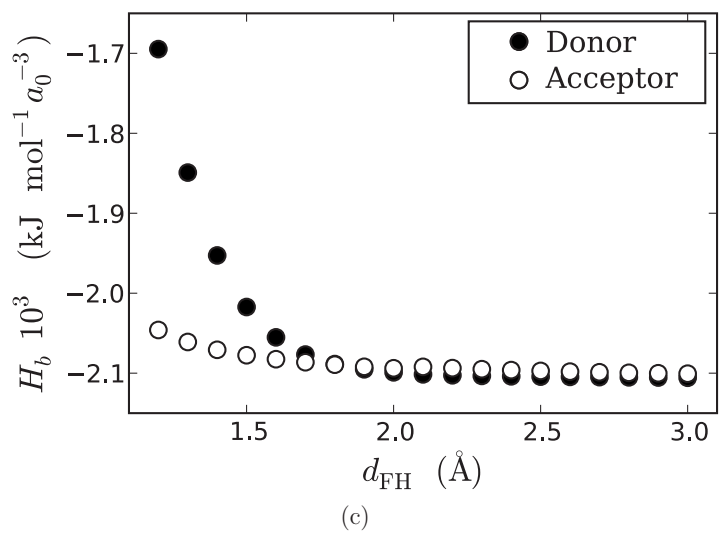

FIG. 4. (a) $\rho_{b}$, (b) $\nabla^{2} \rho_{b}$, and (c ) $H_{b}$ vs $d_{\mathrm{FH}}$ for both covalent bonds in the complex.

respectively, experience the same expansion or contraction when $d_{\mathrm{FH}}$ varies. According to the first term in Eq. (2), this slope increases with $\epsilon$, indicating that if a positive $\epsilon$ is applied, the variation in the size of the fluorine is larger than that of the hydrogen, while a negative $\epsilon$ has the opposite effect. This can be explained by the polarizing effect of the external electric field because the closed shells of the fluorine and hydrogen atoms shift toward and away from the bonding region, respectively, with $\epsilon$. The closed shell polarized toward the bonding region by the electric field is the one that absorbs the variations in the bonding distance, while that of the other atom tends to keep its size.

In the case $\epsilon=0$, the dependencies of $\rho_{b}, \lambda_{\|, b}, \lambda_{\perp, b}, G_{b}$, and $V_{b}$ with $d_{\mathrm{FH}}$ can be represented by exponentials of the form $a e^{-b d_{\mathrm{FH}}}$, the coefficients $a$ and $b$ being determined by a least-squares fitting. For each of these properties, the effect of the external field on these dependencies can be represented by an additional term proportional to $\epsilon$ in the exponential, taking the form

$$
a e^{k \epsilon-b d_{\mathrm{FH}}} \text {. }
$$

The dependence of $H_{b}$ and $\nabla^{2} \rho_{b}$ on $d_{\mathrm{FH}}$ is very similar to the negative of a Morse-type potential, ${ }^{8}$ which is the type of function successfully fitted to $\nabla^{2} \rho_{b}$ for a large set of bonding interactions from experimental electron densities in crystals. ${ }^{35}$ In the linear $(\mathrm{FH})_{2}$ for any given $\epsilon$ value, both $\nabla^{2} \rho_{b}$ and $H_{b}$ can be fitted to a function that results from the addition of two exponentials ${ }^{8}$ and includes the effect of the field,

$$
a_{1} e^{-k \epsilon-b d_{\mathrm{FH}}}-a_{2} e^{k \epsilon-2 b d_{\mathrm{FH}}} .
$$

The parameters $a, a_{1}, a_{2}, b$, and $k$ in Eqs. (3) and (4) have been determined by least-squares fits (Table I and Fig. 5), allowing a description of the dependence of all these properties on $d_{\mathrm{FH}}$ and $\epsilon$. In the case of $\rho_{b}$ and $V_{b}, k$ was set to 0 as these properties do not vary significantly with $\epsilon$.

It must be noticed that in spite of the excellent $R$ factors, these simple fits do not reproduce the detailed features of the dependencies on both $d_{\mathrm{FH}}$ and $\epsilon$. While, according to the fittings, the dependencies on $d_{\mathrm{FH}}$ are exponential at fixed $\epsilon$ values, deviations can be observed for any property, especially at long $d_{\mathrm{FH}}$ distances where their variation with $\epsilon$ is close to zero. Hence, the fits are mainly useful in describing the overall variation of the properties with $\epsilon$ but not in showing the exact dependence observed for each particular value on $\epsilon$.

The formation of the hydrogen bond does not introduce qualitative changes in the MOs of both monomers. ${ }^{36}$ In its fundamental state, each FH has eight valence electrons distributed in a bonding orbital $(2 \sigma)$ and three nonbonding orbitals ( $3 \sigma$ and two degenerate $\pi$ orbitals). While the $2 \sigma$ and $3 \sigma$ orbitals are distributed along the molecule, both $\pi$ orbitals are strongly localized around the fluorine. ${ }^{15,37}$ As $3 \sigma$ is more localized on the fluorine than $2 \sigma$, the closed shells involved in the hydrogen bond are mostly contributed by the $2 \sigma$ orbital of the donor molecule, which is associated with the covalent bond, and by the $3 \sigma$ and $\pi$ orbitals of the acceptor molecule, which mainly correspond to the fluorine lone pairs.

At BCP, while the electric field generated by the nucleus of the acceptor fluorine is completely screened by the electron distribution of this atom, the electric field coming from the hydrogen nucleus stabilizes the electron distribution of the fluorine lone pairs in the bonding region. ${ }^{33}$ Furthermore, the latter field is partly screened by the bonding orbital of the donor molecule, its effective screening being reduced as the positive external field polarizes this orbital away from the bonding region. Accordingly, the external field approaches the BCP toward the hydrogen nucleus of the donor molecule, indicating that the region of $\rho(\mathbf{r})$ that screens this nucleus is reduced. Both effects result in an increase in the total electric field around the $\mathrm{BCP}$ and, in particular, from the directions perpendicular to the bond, pushing the electron density to- 


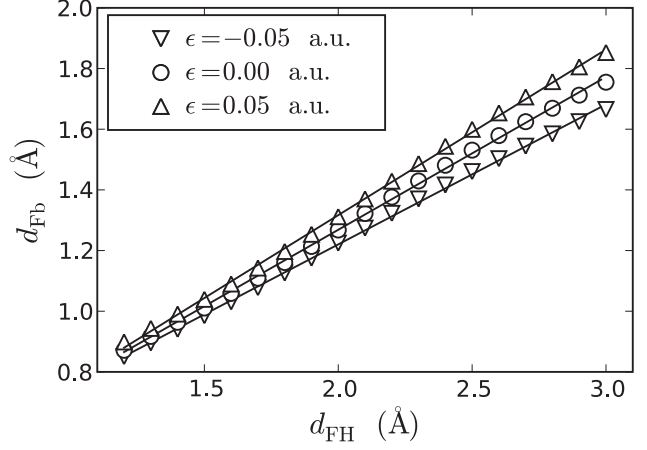

(a)

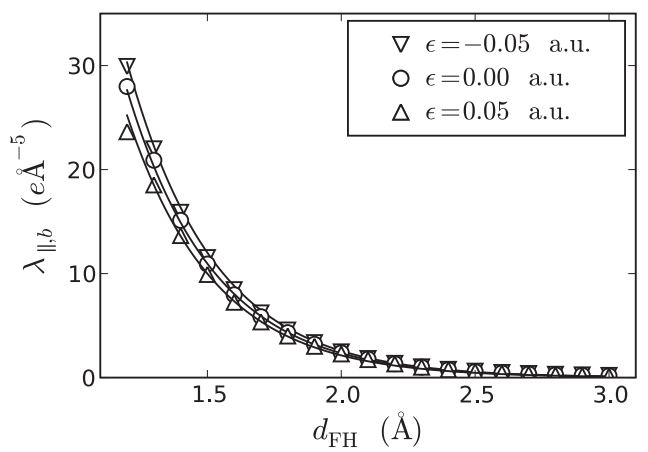

(c)

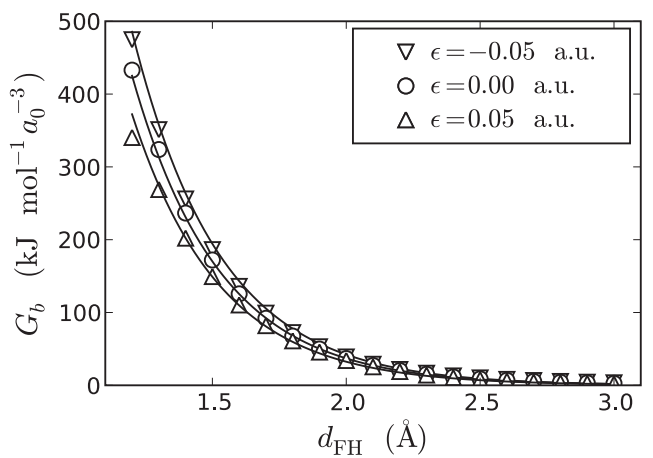

(e)

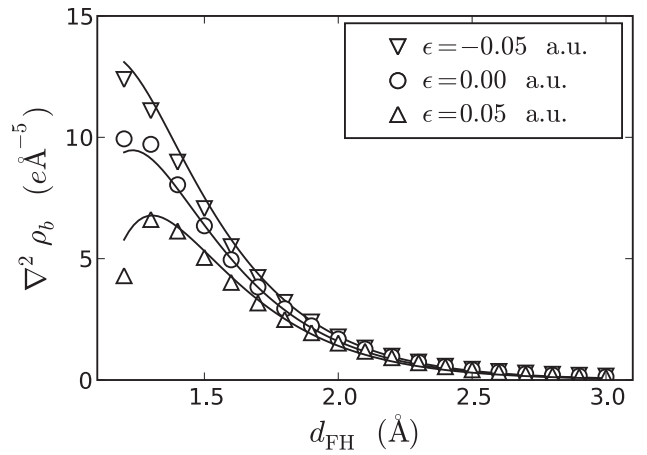

(g)

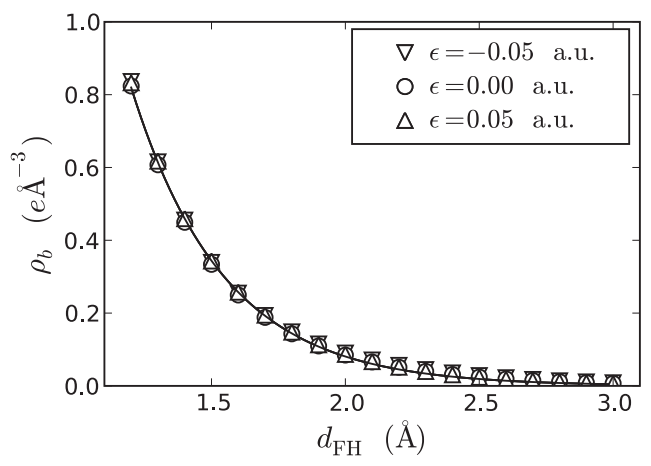

(b)

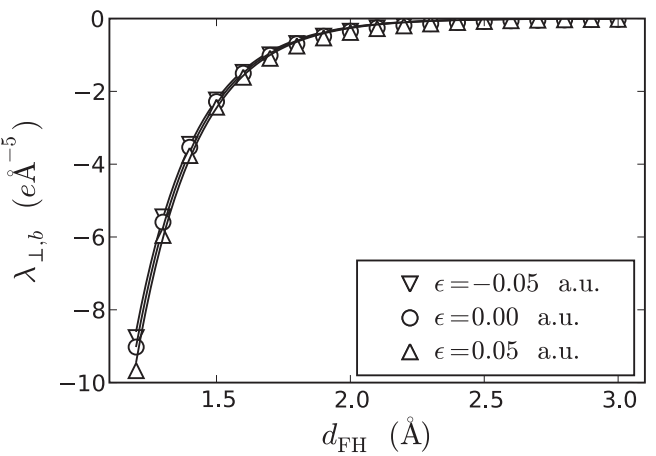

(d)

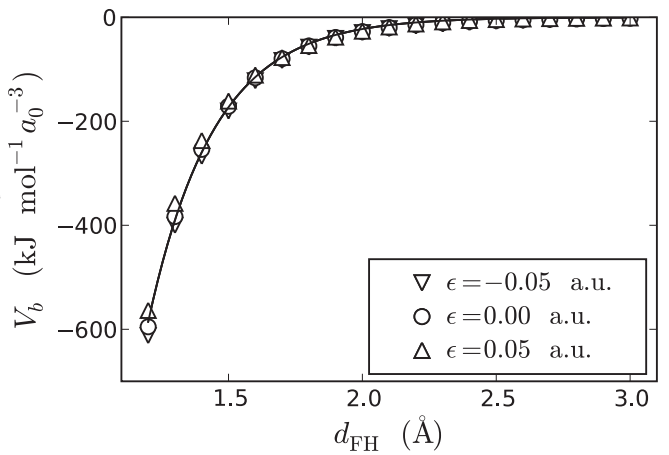

(f)

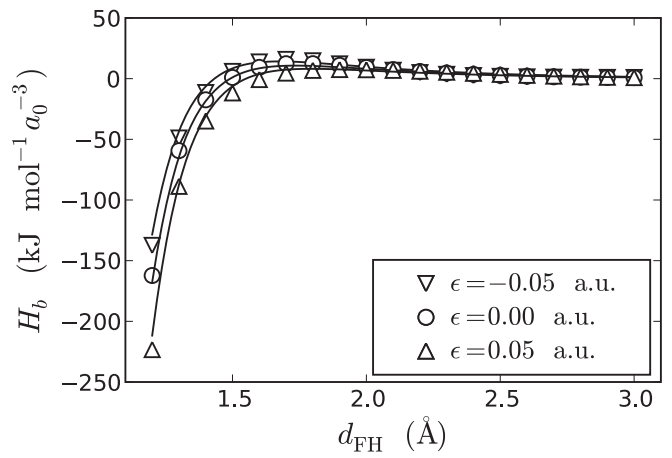

(h)

FIG. 5. (a) $d_{\mathrm{F} b}$, (b) $\rho_{b}$, (c) $\lambda_{\|, b}$, (d) $\lambda_{\perp, b}$, (e) $G_{b}$, (f) $V_{b}$, (g) $\nabla^{2} \rho_{b}$, and (h) $H_{b}$ vs $d_{\mathrm{FH}}$ for $\epsilon=-0.05,0.0$, and 0.05 a.u. Lines correspond to the fitting functions given in Eq. (2) and Table I (Ref. 53).

ward the bond axis and increasing $\left|\lambda_{\perp, b}\right|$. The negative external field has the opposite effect, increasing the region that screens the hydrogen nucleus and reducing the electrostatic pressure from this nucleus on the hydrogen bond, with the concomitant decrease of $\left|\lambda_{\perp, b}\right|$.

As the bonding $\mathrm{MO}$ of the donor is removed from the hydrogen bonding region with $\epsilon>0, \rho(\mathbf{r})$ should decrease here. However, for $\epsilon>0$, the screening of the hydrogen nucleus also decreases, and therefore a stronger electric field in the direction perpendicular to the bond polarizes $\rho(\mathbf{r})$ toward the $\mathrm{H} \cdots \mathrm{F}$ bond path, as observed by the increase in $\left|\lambda_{\perp, b}\right|$ with $\epsilon$. In that way, the variations in $\rho_{b}$ induced by both effects tend to cancel, keeping $\rho_{b}$ approximately constant. This feature is also exhibited in $V_{b}$, which represents 
TABLE I. Fitting parameters of the topological properties at BCP.

\begin{tabular}{|c|c|c|c|c|c|c|}
\hline & Function & \multicolumn{2}{|c|}{$a$} & $b$ & $k$ & $R$ \\
\hline$\rho\left(e \AA^{-3}\right)$ & $a e^{-b d_{\mathrm{FH}}}$ & \multicolumn{2}{|c|}{$26.5(3)$} & $2.897(8)$ & & 0.9998 \\
\hline$\lambda_{\|, b}\left(e \AA^{-5}\right)$ & Eq. (3) & \multicolumn{2}{|c|}{$1121(14)$} & $3.085(9)$ & $-1.84(5)$ & 0.9997 \\
\hline$\lambda_{\perp, b}\left(e \AA^{-5}\right)$ & Eq. (3) & \multicolumn{2}{|c|}{$-1964(47)$} & $4.49(2)$ & $0.95(6)$ & 0.9996 \\
\hline$G_{b}\left(\mathrm{~kJ} \mathrm{~mol}^{-1} a_{0}^{-3}\right)$ & Eq. (3) & \multicolumn{2}{|c|}{$1.66(3) \times 10^{4}$} & $3.05(1)$ & $-2.66(6)$ & 0.9994 \\
\hline \multirow[t]{2}{*}{$V_{b}\left(\mathrm{~kJ} \mathrm{~mol}^{-1} a_{0}^{-3}\right)$} & $a e^{-b d_{\mathrm{FH}}}$ & \multicolumn{2}{|c|}{$-7.72(23) \times 10^{4}$} & $4.07(2)$ & & 0.9991 \\
\hline & & $a_{1}$ & $a_{2}$ & $b$ & $k$ & $R$ \\
\hline$\nabla^{2} \rho_{b}\left(e \AA^{-5}\right)$ & Eq. (4) & $937(41)$ & $2.32(10) \times 10^{4}$ & $3.17(3)$ & $2.24(5)$ & 0.9981 \\
\hline$H_{b}\left(\mathrm{~kJ} \mathrm{~mol}^{-1} a_{0}^{-3}\right)$ & Eq. (4) & $5.0(2) \times 10^{3}$ & $5.2(4) \times 10^{5}$ & $3.13(3)$ & $1.97(5)$ & 0.9984 \\
\hline
\end{tabular}

the pressure of the system on the electrons in the bonding region, as this quantity remains approximatively constant with the variations in the external field.

The decrease in both $\lambda_{\|, b}$ and $G_{b}$ with positive $\epsilon$ is related to a weakening of the Pauli repulsion between the closed shells, which can be explained by a larger polarizability of the MO associated with the covalent bond than those associated with the lone pairs. Thus, the positive field displaces the $\mathrm{MO}$ of the donor molecule from the bonding region, decreasing the Pauli repulsion. Reversely, the negative field pushes this $\mathrm{MO}$ against the lone pairs of the acceptor fluorine, increasing the Pauli repulsion and therefore the positive magnitudes of $\lambda_{\|, b}$ and $G_{b}$.

The decrease in $\lambda_{\|, b}$ and the increase in $\left|\lambda_{\perp, b}\right|$ with $\epsilon$ contrast with the increase in both $\lambda_{\|, b}$ and $\left|\lambda_{\perp, b}\right|$ when the molecules approach. This is explained by qualitatively different effects on the MOs in both cases. In particular, when molecules approach, both closed shells are brought together and the electron density around BCP becomes steeper in all directions, while the polarization of the donor bonding orbital induced by $\epsilon$ makes the electron density flatter along the bond direction.

According to Eq. (4), both $\nabla^{2} \rho_{b}$ and $H_{b}$ can be represented by the sum of two exponentials with different signs. The positive term is associated with the Pauli repulsion between the closed shells, and it is related to the long-range behavior of both quantities, while the second one dominates at short distances and is related to the stabilization of the electron density in the bonding region induced by the hydrogen nucleus. The positive term decreases with $\epsilon$ as the Pauli repulsion between the closed shells weakens, while the negative term increases in absolute value with $\epsilon$ as the descreening of the hydrogen nucleus strengthens its electric field in the hydrogen bond. The overall effect is the decrease in both $H_{b}$ and $\nabla^{2} \rho_{b}$ with $\epsilon>0$, indicating a net stabilization of the electrons in the bonding region. This effect favors the transition from a closed-shell interaction to a covalent bond and is observed on the shift to larger distances of the geometry corresponding to $H_{b}=0$, which represents the shortest $d_{\mathrm{FH}}$ for a pure closed-shell interaction. ${ }^{14}$ Moreover, the first welldefined local maximum of $\nabla^{2} \rho_{b}$ at longer distances is observed for the strongest positive field, a feature also associated with the transition from closed-shell to shared-shell interactions.

The effect of the positive field on the dependence of $H_{b}$ and $\nabla^{2} \rho_{b}$ on $d_{\mathrm{FH}}$ is qualitatively similar to that observed when removing one electron from the isolated $\mathrm{F} \cdots \mathrm{H}$ pairwise interaction. ${ }^{15}$ Moreover, the effect of the negative field on the same properties is similar to the addition of one electron to the isolated $\mathrm{F} \cdots \mathrm{H}$ system. Thus, as similarly observed in the negative ionization of the $\mathrm{F} \cdots \mathrm{H}$ system, which increases the Pauli repulsion between the closed shells of both atoms, the negative field applied to $(\mathrm{FH})_{2}$ leads to the significant rise in $G_{b}$. In the $\mathrm{F} \cdots \mathrm{H}$ system, this is explained by the repulsion between the former charge in the bonding region and the added electron, which actually occupies the $4 \sigma$ antibonding orbital even at relatively long $d_{\mathrm{FH}}$ distances (for long distances it mainly belongs to the F-atom lone pairs). This stronger interaction pushes the closed shells of the atoms against each other, increasing the Pauli repulsion.

In the positive ionization of $\mathrm{F} \cdot \mathrm{H}$, the electron is removed from the F-atom lone pairs at short $d_{\mathrm{FH}}$ distances and from the $\mathrm{H}$-atom at long $d_{\mathrm{FH}}$ distances. While at short distances the effect is opposite to adding one electron, i.e., the Pauli repulsion between closed shells relaxes because of the weaker effect of the F-atom lone pairs, for long distances there is no Pauli repulsion as the $\mathrm{H}$-atom has formally no electrons. Thus, $(\mathrm{F} \cdots \mathrm{H})^{+}$represents an extreme case in which there is no Pauli repulsion hindering the formation of the bonding orbital. In a hydrogen bond, this could be considered as equivalent to a very large deformation of the hydrogen atom electron distribution away from the bonding region, which can be assimilated to the polarization induced by a very large positive field.

\section{The effect of the external electric field on the integrated properties}

The most important effect of the external electric field on the MOs is the polarization of the bonding orbital in both FH molecules. The positive field polarizes these orbitals toward both fluorine atoms, increasing their atomic charge and stabilizing the molecules for all $d_{\mathrm{FH}}$ distances. A similar effect has been observed on the atomic charges of the $\mathrm{H}_{3} \mathrm{CH} \cdots \mathrm{OH}_{2}$ complex upon application of an electric field. ${ }^{38}$ The redistribution of the charge in the molecules affects the intermolecular interaction, leading to modifications in the integrated properties that depend on $d_{\mathrm{FH}}$. Thus, while the positive field shifts the integrated energy of the dimer $(E)$ to more negative values (Fig. 6(a)), the same is observed for the 


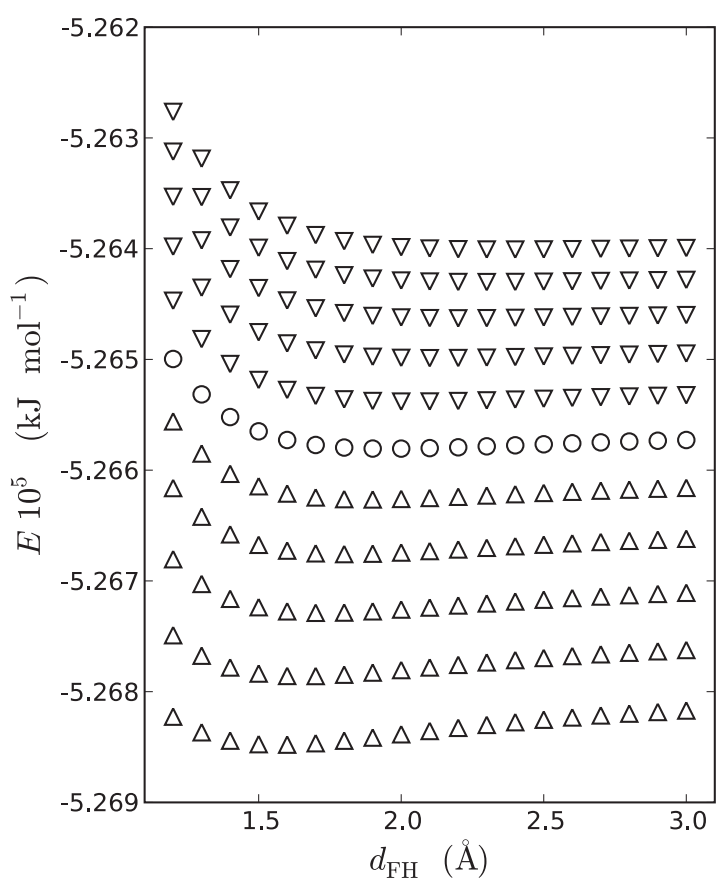

(a)

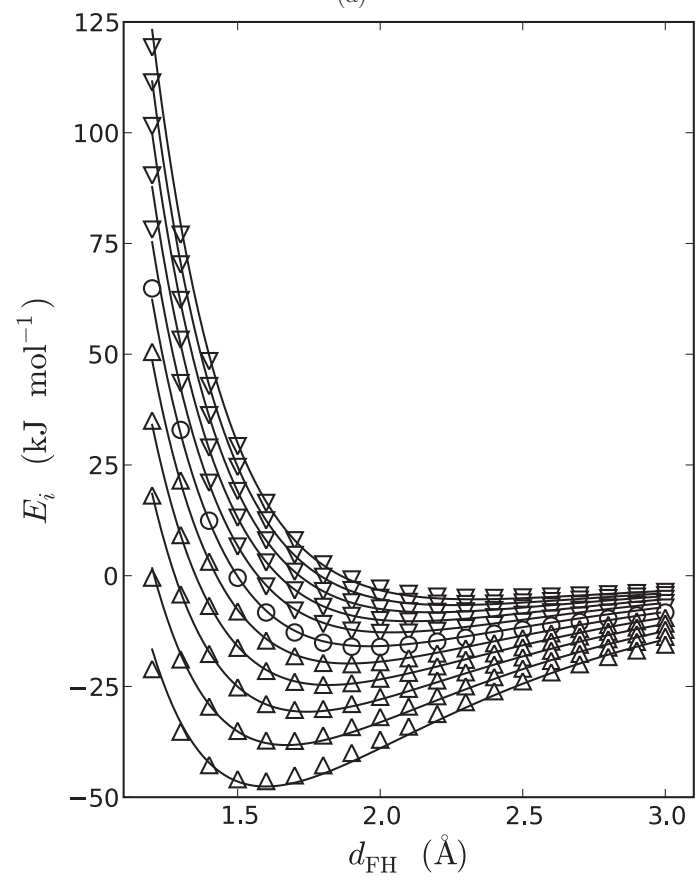

(b)

FIG. 6. (a) Total energy $(E)$ and (b) interaction energy $\left(E_{i}\right)$ of the dimer Down triangles, circles, and up triangles correspond to $\epsilon<0, \epsilon=0$, and $\epsilon>0$, respectively. Lines in (b) correspond to the fittings obtained from Eq. (7) (Ref. 53).

interaction energy $E_{i}$, defined as the energy of the complex at a fixed $\epsilon$ field minus the energy of the isolated monomers $\left(E_{\mathrm{FH}}\right)$ for the same $\epsilon$ value (Fig. 6(b)),

$$
E_{i}\left(d_{\mathrm{FH}}, \epsilon\right)=E\left(d_{\mathrm{FH}}, \epsilon\right)-2 E_{\mathrm{FH}}(\epsilon) .
$$

In order to explore the relationship between the interaction energy of the complex and the electron density properties at the hydrogen bond BCP, the dependence of the interaction energy on both $d_{\mathrm{FH}}$ and $\epsilon$ has been fitted to a sum of two exponentials, similar to the form given in Eq. (3),

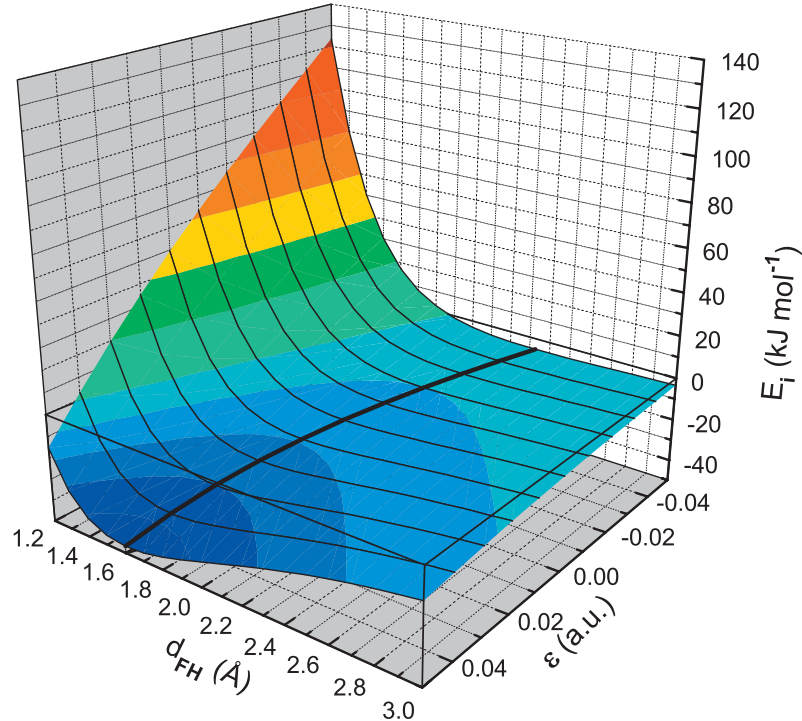

FIG. 7. (Color) Interaction energy surface $E_{i}\left(d_{\mathrm{FH}}, \epsilon\right)$ from Eq. (7). Thick line crossing the surface corresponds to the dependence of $E_{i}$ at the equilibrium distance $d_{\mathrm{eq}}$ on the applied field $\epsilon\left[E_{i}\left(d_{\mathrm{eq}}(\epsilon), \epsilon\right)\right.$ with $d_{\mathrm{eq}}(\epsilon)$ from Eq. (9), or $E_{i}\left(d_{\mathrm{eq}}, \epsilon\left(d_{\mathrm{eq}}\right)\right)$ with $\epsilon\left(d_{\mathrm{eq}}\right)$ from Eq. (11)]. Thin lines along $d_{\mathrm{FH}}$ correspond to the $E_{i}$ dependence on $d_{\mathrm{FH}}$ for each applied field. Colors are set each at $20 \mathrm{~kJ} \mathrm{~mol}^{-1}$ for $E_{i}>0$ and each at $10 \mathrm{~kJ} \mathrm{~mol}^{-1}$ for $E_{i}<0$.

$$
E_{i}\left(d_{\mathrm{FH}}, \epsilon\right)=-a_{1} e^{k_{1} \epsilon-b_{1} d_{\mathrm{FH}}}+a_{2} e^{-k_{2} \epsilon-b_{2} d_{\mathrm{FH}}},
$$

giving

$$
\begin{aligned}
E_{i}\left(d_{\mathrm{FH}}, \epsilon\right)= & -193(9) e^{13.9(2) \epsilon-1.10(2) d_{\mathrm{FH}}} \\
& +1.02(4) 10^{4} e^{-5.3(2) \epsilon-3.74(4) d_{\mathrm{FH}}},
\end{aligned}
$$

with $R=0.9994$ (Fig. 7).

The dependence of the interaction energy on the external field presents a minimum for all the values of $\epsilon$. The minimum is displaced to larger distances and becomes shallower for negative fields. As far as the interaction remains as a closed-shell type, the stabilization induced by the mutual polarization is expected to extend along the complete range of the considered distances in spite of the incipient formation of the bonding orbital at shorter distances. The polarization effect is thus superimposed to that of the reorganization of the MOs in the integrated atomic energies. Then, as the positive field strengthens the mutual polarization, the shortest $d_{\mathrm{FH}}$ for which this effect represents the main contribution to the interaction energy moves to shorter distances with $\epsilon$.

The effect of the mutual polarization can be observed on the contributions of the individual atoms to the interaction energy (Figs. 3(b) and 8). In the region where the interaction is dominated by the mutual polarization $\left(d_{\mathrm{FH}} \geqslant 2.0 \AA\right.$ for $\epsilon$ $=0.05$ a.u. and $d_{\mathrm{FH}} \gtrsim 2.3 \AA$ for $\epsilon=-0.05$ a.u.), a stabilization of both F-atoms and a destabilization of both $\mathrm{H}$-atoms are exhibited as $d_{\mathrm{FH}}$ is reduced. This region is clearly larger at $\epsilon=0.05$ a.u. than at $\epsilon=0.0$ a.u., and is hardly visible at $\epsilon=-0.05$ a.u. because of the smaller effect of the mutual polarization in the donor molecule. According to this interpretation, the shift in the interaction energy minimum toward lower energies and shorter $d_{\mathrm{FH}}$ with the positive field is related to an enhancement of the electrostatic interaction.

The weakening of the covalent bonds in both donor and acceptor molecules is observed on the topological and ener- 


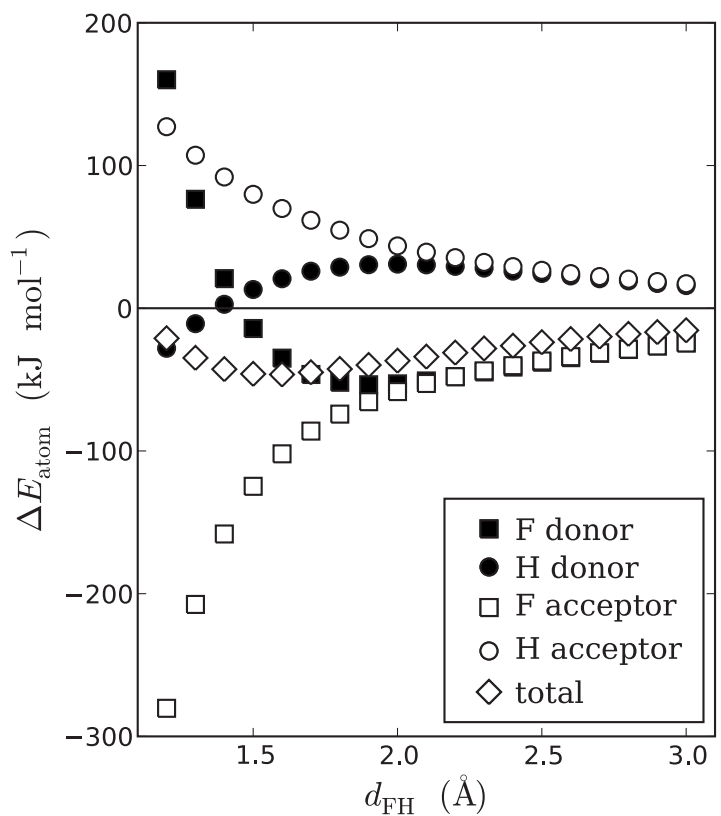

(a)

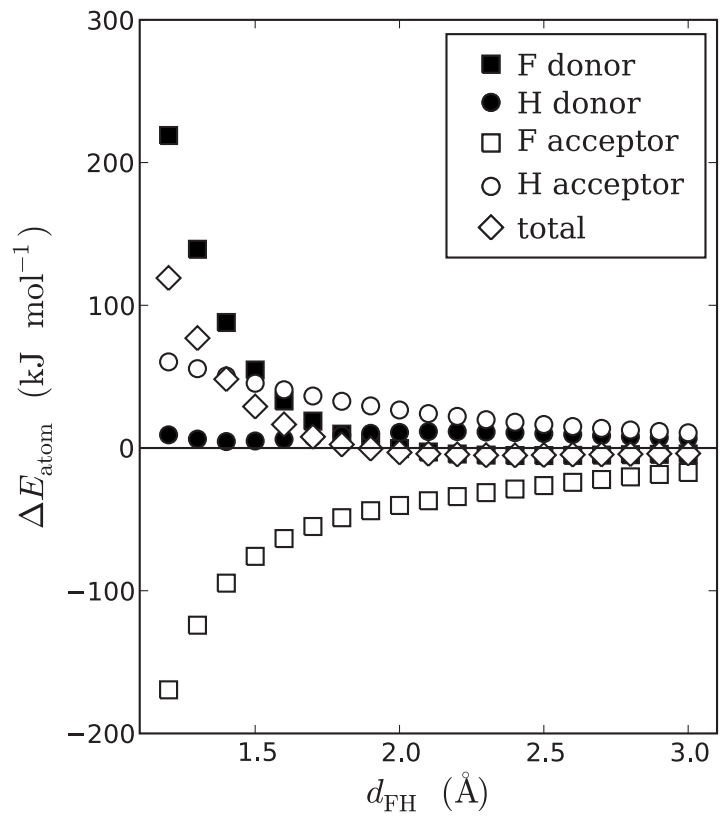

(b)

FIG. 8. Variation in the atomic energies with respect to the monomer upon the same external field. $\left(\Delta E_{\text {atom }}\right)$ vs $d_{\mathrm{FH}}$ for (a) $\epsilon=0.05$ a.u. and (b) $\epsilon=-0.05$ a.u.

getic properties at the corresponding BCPs. Thus, above a threshold value of $d_{\mathrm{FH}}$ that depends on the external field, all the BCP properties of the covalent bonds remain constant with the variation in $d_{\mathrm{FH}}$. Below this threshold, the transformation of the covalent bond starts, as shown by the decreasing $\rho_{b}$ (Fig. 9(a)) and $\left|V_{b}\right| / G_{b}$ (Fig. 9(b)), which evolve toward the range of values expected for a closed-shell interaction. While the threshold shifts to larger $d_{\mathrm{FH}}$ with $\epsilon$, the rise in the energy of the fluorine in the donor molecule starts at shorter $d_{\mathrm{FH}}$ because of the effect of the mutual polarization in the integrated properties.

While the topological analysis of $\rho(\mathbf{r})$ indicates that the

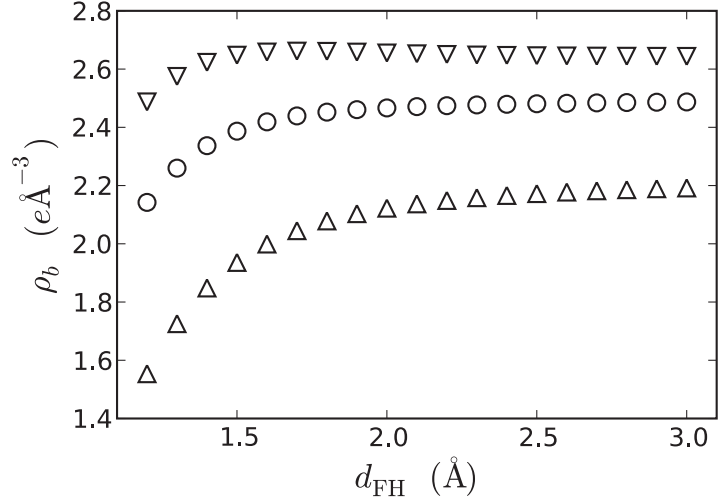

(a)

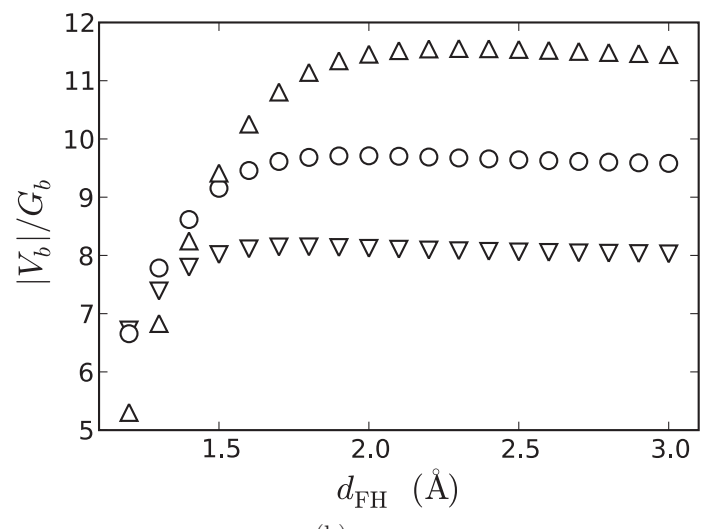

(b)

FIG. 9. (a) $\rho_{b}$ and (b) $\left|V_{b}\right| / G_{b}$ at the BCP of the donor molecule covalent bond vs $d_{\mathrm{FH}}$. Up triangles, circles, and down triangles correspond to $\epsilon=-0.05,0$, and 0.05 a.u., respectively.

negative and positive exponentials in Eqs. (6) and (7) can be, respectively, associated with the mutual polarization and with the reorganization of the MOs involved in the transition from the hydrogen bond to the covalent bond, there is no clear correspondence between these two contributions to the interaction energy and the energy components defined in conventional energy decomposition methods. ${ }^{39}$ However it is reasonable to assume that the mutual polarization can be mainly associated with electrostatic and induction energies, as both of them are more important for long-range distances. This is not in contradiction with the common view of the hydrogen bond interaction, considered as mainly electrostatic in spite of the important quantic effects. Indeed, the key role of the mutual polarization stresses the importance of the induction energy in tuning the properties of the hydrogen bond, as the effect of the external electric field is more important for the long-range than for the short-range components of the interaction energy $\left[k_{1}>k_{2}\right.$ in Eq. (6)]. This result agrees with the correlation between the delocalization energy, which is also related to the mutual polarization, and the bonding distance observed in resonance assisted hydrogen bonds, ${ }^{40}$ which are characterized by a high polarizability of the MOs involved.

While mutual polarization can be traced back to the electrostatic and induction energies, the relationship between the MO reorganization and the short-range energy contributions, such as charge transfer and exchange repulsion, is more com- 


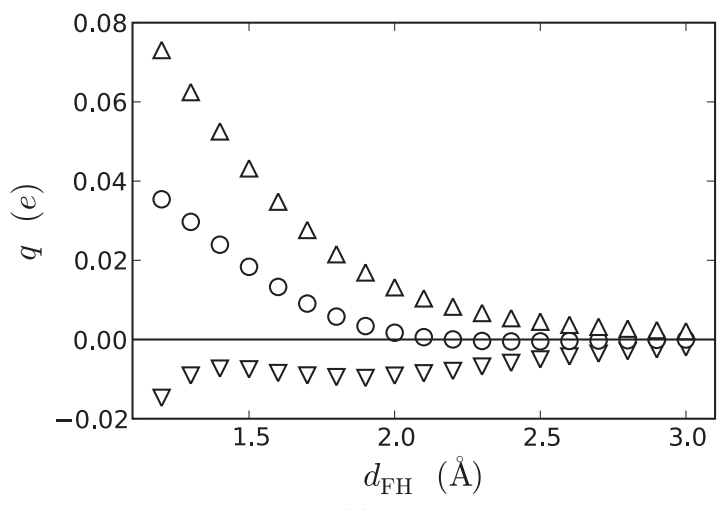

(a)

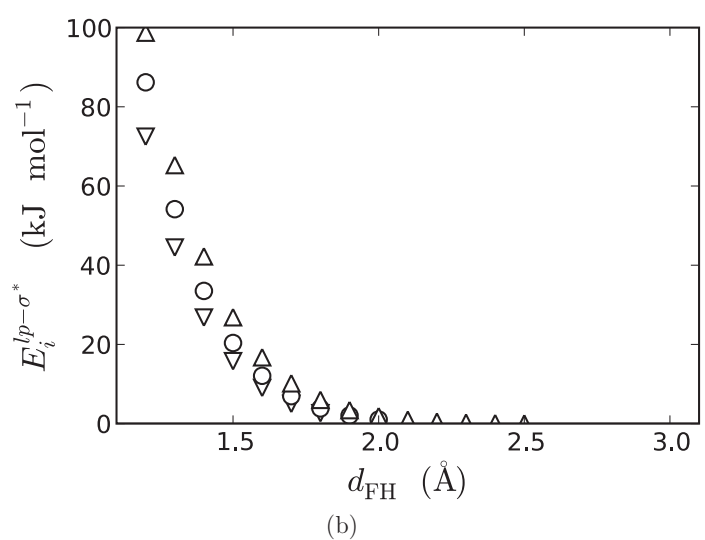

FIG. 10. (a) Net charge $(q)$ of the acceptor molecule and (b) interaction energy $E_{i}^{l p \rightarrow \sigma^{*}}$ between the lone pair in the acceptor molecule and the antibonding orbital of the donor molecule. Symbols are defined as in Fig. 9.

plex. The exchange-repulsion component, which is related to Pauli's exclusion principle, is usually associated with the energy rise at short distances in pairwise atom-atom interaction potentials. However, this contribution seems to play a secondary role in Eq. (6), as shown by the stabilization of the central atoms of the complex (Fig. 8) at short distances or by the behavior of the $\mathrm{BCP}$ properties, suggesting a relaxation of the Pauli repulsion as the orbital reorganization takes place.

Charge transfer can be calculated by charge integration on the atomic basins according to QTAIM (Fig. 10(a)). Both the weaker Pauli repulsion and the induced polarization of the orbitals favor the electron transfer from the acceptor to the donor molecules, and therefore charge transfer starts at longer distances and is larger with the positive external field. The negative field has the opposite effect and can even reverse the sign of the charge transfer. Accordingly, for the strongest negative field, the acceptor molecule is negatively charged within the whole $d_{\mathrm{FH}}$ range.

The energy of the charge transfer from the acceptor fluorine lone pair to the donor antibonding orbital $\left(E_{i}^{l p \rightarrow \sigma^{*}}\right)$, as calculated by the NBO analysis, ${ }^{41}$ gives similar results for all external applied fields regardless of the sign of the charge transfer calculated according to QTAIM. $E_{i}^{l p \rightarrow \sigma^{*}}$ shows a similar dependence on $d_{\mathrm{FH}}$ for all the applied fields, although this interaction is weaker and takes place in a shorter range of $d_{\mathrm{FH}}$ as the field is more negative (Fig. 10(b)). Charge transfer appears as a short-range stabilizing contribution to
$E_{i}$, related to both the incipient covalent bond formation and the stabilization of the atoms in the hydrogen bond. However, this is only part of the MO reorganization that extends beyond the hydrogen bonding region and has an overall destabilizing effect.

The positive and negative values of the exponents $k_{1} \epsilon$ and $-k_{2} \epsilon$ in Eq. (6), respectively, show that both long- and short-range contributions to the interaction energy decrease with $\epsilon$. Thus, the complex is stabilized with $\epsilon$ in the whole range of $d_{\mathrm{FH}}$ distances, regardless of which contribution dominates $E_{i}$. In that way, the strengthening of the mutual polarization and the smaller destabilization of the molecular covalent bond, both of them induced by $\epsilon>0$, show as the shortening of the equilibrium distance in Eq. (6). Therefore, the stabilization of the complex induced by the external field is accompanied by an approaching of the donor and acceptor molecules if the $d_{\mathrm{FH}}$ distance is allowed to relax.

The analysis of the integrated properties demonstrates that the effect of the external field on the interaction energy is explained by either the enhancement $(\epsilon>0)$ or the weakening $(\epsilon<0)$ of the electrostatic interaction, which is reflected by the mutual polarization of the molecules. Although the concomitant reorganization of the MOs plays a fundamental role in the interaction energy at short $d_{\mathrm{FH}}$, the most important changes induced by the external field take place on the electrostatic part of the interaction, conditioning the form of the interaction energy curve.

Even though several results suggest that there is a close relationship between local (at BCP) and global (integrated) properties, ${ }^{2}$ the rationalization of these properties in terms of the MOs involved in the interaction stresses the complexity of this relationship. In spite of the similarity of both $H_{b}$ and $E_{i}$ dependencies on $d_{\mathrm{FH}},{ }^{14}$ these properties are actually showing different aspects from the same interaction. Thus, while the $H_{b}$ dependence on $d_{\mathrm{FH}}$ is controlled by the incipient covalent bond at short $d_{\mathrm{FH}}$ distances, and by the Pauli repulsion at long $d_{\mathrm{FH}}$ distances, $E_{i}$ is governed by the weakening of the covalent bonds in the interacting molecules and by the mutual polarization between both molecules at short and long $d_{\mathrm{FH}}$ distances, respectively. The relationship between the position of the maximum of $H_{b}$ and the minimum of $E_{i}$ in the dependencies of both quantities on $d_{\mathrm{FH}}$ appears related to the starting reorganization of the bonding orbital in the hydrogen bonding region.

Moreover, while the effect of the external field on the integrated properties can be mainly explained from the electrostatic part of the interaction, the effect on the critical point properties at the hydrogen bond $\mathrm{BCP}$ is mostly related to the Pauli repulsion between the electron closed shells. In this way, the electric field has a different effect on both kinds of properties. For example, the positive field strengthens the electrostatic interaction, displacing the equilibrium distance to shorter $d_{\mathrm{FH}}$, but it also weakens the Pauli repulsion at the hydrogen bond, shifting the maxima of $H_{b}$ and $\nabla^{2} \rho_{b}$ to larger $d_{\mathrm{FH}}$.

\section{BCP properties at the equilibrium geometry}

In most cases, topological properties are evaluated in families of complexes $\mathrm{X}-\mathrm{D}-\mathrm{H} \cdots \mathrm{A}-\mathrm{Y}$, where $\mathrm{A}$ and $\mathrm{D}$ are 
identical in all the family members while $\mathrm{X}$ and $\mathrm{Y}$ are different. For each complex, BCP properties are usually evaluated at the relaxed geometry, which corresponds to the $\mathrm{H} \cdots \mathrm{A}$ equilibrium distance. Thus, for each BCP property, the family of complexes has associated the dependence on the $\mathrm{H} \cdots \mathrm{A}$ bonding distance, built from the calculated values at the equilibrium geometries of the members.

A further procedure can be followed by considering each complex of the family independently of the others. Here, the topological properties can be evaluated at $\mathrm{H} \cdots \mathrm{A}$ distances other than the equilibrium one, giving rise to a set of dependencies on the bonding distance that represents a reaction coordinate for the chosen complex.

Accordingly, two kinds of dependencies can be obtained for a family of complexes: the equilibrium dependencies, characteristic of the family, and the reaction coordinate dependencies, characteristic of each complex. If the complexes in the family are similar enough, their respective reaction coordinate dependencies should be roughly similar and, in addition, close to the equilibrium dependencies. In this case, $\mathrm{X}$ and $\mathrm{Y}$ can be considered as a perturbation on the $\mathrm{H} \cdots \mathrm{A}$ interaction that will exhibit as a slightly different set of reaction coordinate dependencies for each member of the family.

In the linear $(\mathrm{FH})_{2}$, the effect of the external electric field on the hydrogen bond is explained by the polarization of the MOs involved in the interaction. It can be supposed that this polarization is not qualitatively different from that induced by the chemical environment, namely, the $\mathrm{X}$ and $\mathrm{Y}$ substituents in the set of $\mathrm{X}-\mathrm{D}-\mathrm{H} \cdots \mathrm{A}-\mathrm{Y}$ complexes. In this way, the linear dimer $(\mathrm{FH})_{2}$ upon applied external field can be considered as similar to a family of complexes, each member being identified by an effective polarization $\epsilon$. Hence, the perturbation previously represented by $\mathrm{X}$ and $\mathrm{Y}$ is actually interpreted in terms of the value of $\epsilon$. The main difference between both cases comes from the fact that while in a set of $\mathrm{X}-\mathrm{D}-\mathrm{H} \cdots \mathrm{A}-\mathrm{Y}$ complexes the change in $\mathrm{X}$ and $\mathrm{Y}$ corresponds to discrete modifications on the perturbation, in the case of $(\mathrm{FH})_{2}$ it can be continuously tuned by means of $\epsilon$.

For example, in the study of the $\mathrm{XH} \cdots \mathrm{CNH}$ family of complexes, ${ }^{42}$ the change in $\mathrm{X}$ can be considered to play a role similar to the external $\epsilon$ field in $(\mathrm{FH})_{2}$ because the increase in the electronegativity of $\mathrm{X}$ has roughly the same effect than a positive external field. Indeed, in that case, the closed shell of the donor molecule withdraws from the bonding region, favoring shorter bonding distances and larger interaction energies. However, it should be noticed that due to the diversity of substituents taken into account, the effect of the modification of $\mathrm{X}$ is more complex than the simple picture given here. Therefore, what in $(\mathrm{FH})_{2}$ exhibits as linear or exponential dependencies, in the $\mathrm{XH} \cdots \mathrm{CNH}$ family appears as rough correlations. Other families of complexes exhibit similar behaviors, for example, the complexes of $\mathrm{HCl}$ with 4 -X-pyridine, ${ }^{43}$ with the difference that in this last case the substituents are in the acceptor and their effect is so similar that a clear correlation between the interaction energy and the $\mathrm{H} \cdots \mathrm{N}$ distance is observed. Another interesting example is the edge-to-face interaction between aromatic rings, where the substituent effect is explained in terms of electrostatic and polarization effects that depend on the electronegativity of the substituent. ${ }^{44}$ Those examples suggest that the external field can just be taken as a parameter related to a perturbation that polarizes the closed shells of the interacting molecules. This effect can be considered at the origin of the charge transfer from these closed shells. Accordingly, an external field large enough applied to $\mathrm{ClH} \cdots \mathrm{NH}_{3}$ can induce a proton transfer ${ }^{45}$ similar to that induced by the substituent in complexes of $\mathrm{HCl}$ with 4 -X-pyridine. ${ }^{43}$

The effect of the electric field on the equilibrium distance can be determined by solving the equation

$$
\left.\frac{\partial E}{\partial d_{\mathrm{FH}}}\right|_{\epsilon}=\left.\frac{\partial E_{i}}{\partial d_{\mathrm{FH}}}\right|_{\epsilon}=0 .
$$

According to the functional used for $E_{i}$ [Eq. (6)], the following relationship is found between the equilibrium distance $\left(d_{\mathrm{eq}}\right)$ and $\epsilon$ :

$$
d_{\mathrm{eq}}=d_{0}-\frac{k_{1}+k_{2}}{b_{2}-b_{1}} \epsilon,
$$

where

$$
d_{0}=\frac{1}{b_{2}-b_{1}} \ln \frac{a_{2} b_{2}}{a_{1} b_{1}}
$$

represents the equilibrium distance at $\epsilon=0\left[d_{0}=1.966 \AA\right.$ from the fitting parameters, while the observed distance for the relaxed linear $(\mathrm{FH})_{2}$ dimer without an applied external field is $\left.d_{0}=1.955 \AA\right]$. The linear dependence of Eq. (9) can be easily inverted, leading to

$$
\epsilon=\frac{b_{2}-b_{1}}{k_{1}+k_{2}}\left(d_{0}-d_{\mathrm{eq}}\right) \text {. }
$$

Following this methodology, at the equilibrium geometry resulting from each $\epsilon$ perturbation [Eq. (9)], the value of any $\rho(\mathbf{r})$ property at BCP is obtained by substituting Eq. (11) and setting $d_{\mathrm{FH}}=d_{\mathrm{eq}}$ in the corresponding fitting function given in Table I. This function, which is obtained from the dependence of the property on the $\mathrm{H} \cdots \mathrm{A}$ reaction coordinate, permits us thus to retrieve the actual property dependence on the equilibrium distance that the system exhibits as a function of $\epsilon$.

For the values of $\epsilon$ considered here $(-0.05$ a.u. $<\epsilon$ $<0.05$ a.u.) and according to Eq. (9), the equilibrium distances extend along the range 1.60(3) $\AA<d_{\mathrm{FH}}<2.33(4) \AA$, which is very similar to the range of $d_{\mathrm{FH}}$ distances observed for the relaxed linear $\mathrm{FH} \cdots \mathrm{FH}$ dimer upon applied external field $\left(1.568 \AA<d_{\mathrm{FH}}<2.367 \AA\right)$.

If coordinate reaction and equilibrium dependencies are represented for one of the topological properties (for example, $G_{b}$ in Fig. 11), the function corresponding to equilibrium distances appears as a curve that crosses at a single point for each coordinate reaction function (calculated for a given $\epsilon$ ). In that way, the crossing point indicates the $d_{\mathrm{FH}}$ distance that corresponds to the equilibrium configuration of the system for that $\epsilon$ field. It should be noted that in the case 


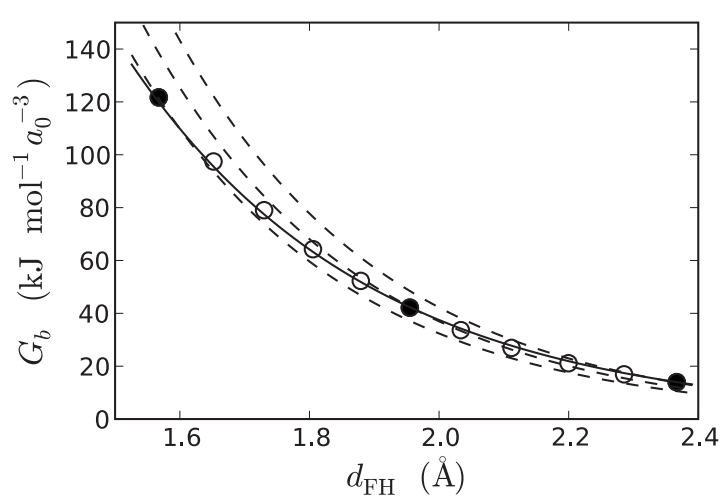

FIG. 11. $G_{b}$ dependence on the equilibrium distance $d_{\mathrm{FH}}=d_{\mathrm{eq}}(\epsilon)$ obtained from Eq. (9) varying $\epsilon$ within the range of $-0.05<\epsilon<0.05$ a.u. (solid line) and on the reaction coordinate distance at $\epsilon=-0.05,0$, and 0.05 a.u. (upper, middle, and lower dashed lines), respectively. Circles correspond to the relaxed dimer at each external electric field $\epsilon$, comparing very nicely to the solid line. Black symbols correspond to circles at $\epsilon=-0.05,0$, and 0.05 a.u.

of a set of $\mathrm{X}-\mathrm{D}-\mathrm{H} \cdots \mathrm{A}-\mathrm{Y}$ complexes, each pair of $\mathrm{X}$ and $\mathrm{Y}$ substituents corresponds to a different perturbation on the $\mathrm{H} \cdots \mathrm{A}$ interaction, in contrast to the tuning of a single perturbation parametrized by $\epsilon$ in the linear $(\mathrm{FH})_{2}$. Thus, the values of the topological and energetic BCP properties at the equilibrium geometries of the $\mathrm{X}-\mathrm{D}-\mathrm{H} \cdots \mathrm{A}-\mathrm{Y}$ complexes do not necessarily fall within the equilibrium dependence curve, as those of the linear $(\mathrm{FH})_{2}$ with the applied external field do, giving rise to the scattering of values around the equilibrium dependencies usually observed when representing the topological properties as a function of the $\mathrm{H} \cdots$ A distance (see, for instance, Ref. 14).

As a simple example of the similarity between the $\rho(\mathbf{r})$ BCP properties observed for a family of complexes $\mathrm{X}-\mathrm{D}-\mathrm{H} \cdot \mathrm{A}-\mathrm{Y}$ and those observed for a single similar system with applied field, we have compared the $a b$ initio calculations carried out for a set of $11 \mathrm{FH} \cdots \mathrm{FY}$ complexes at their equilibrium geometry ${ }^{14}$ to those undertaken for the linear $(\mathrm{FH})_{2}$ dimer. For each complex of the family, an effective electric field was calculated from the equilibrium distance [Eq. (11)] and included in the dependencies given in Table I. The equilibrium distances and the effective fields range from $d_{\mathrm{eq}}=1.732 \AA$ and $\epsilon=0.032$ a.u. (for $\mathrm{Y}=\mathrm{Al}$ ) to $d_{\mathrm{eq}}$ $=2.217 \AA$ and $\epsilon=-0.0345$ a.u. for $(\mathrm{Y}=\mathrm{F})$, falling inside the observed $d_{\mathrm{eq}}$ and the used $\epsilon$ ranges for the linear dimer $(\mathrm{FH})_{2}$. As seen in Fig. 12(a), the values of $\nabla^{2} \rho_{b}$ estimated from the effective $\epsilon$ are almost identical to the results of the $a b$ initio calculations, indicating the equivalence between the perturbation induced by the substituent $\mathrm{Y}$ and that exerted by the external electric field. The linear fit shown in Fig. 12(a) for $\nabla^{2} \rho_{b}$ is given in Table II, along with similar fits for all the other properties gathered in Table I. According to Table II, the result obtained for $\nabla^{2} \rho_{b}$ can be generalized to all topological and energetic properties at the hydrogen bond CP but $H_{b}$ (Fig. 12(b)), for which the values calculated from $\epsilon$ are only roughly proportional to the $a b$ initio magnitudes. The largest deviation from this rough proportionality is found for $\mathrm{FH} \cdots \mathrm{FAl}$, which is the complex with the shortest equilibrium distance.

According to these comparisons, the BCP properties es-

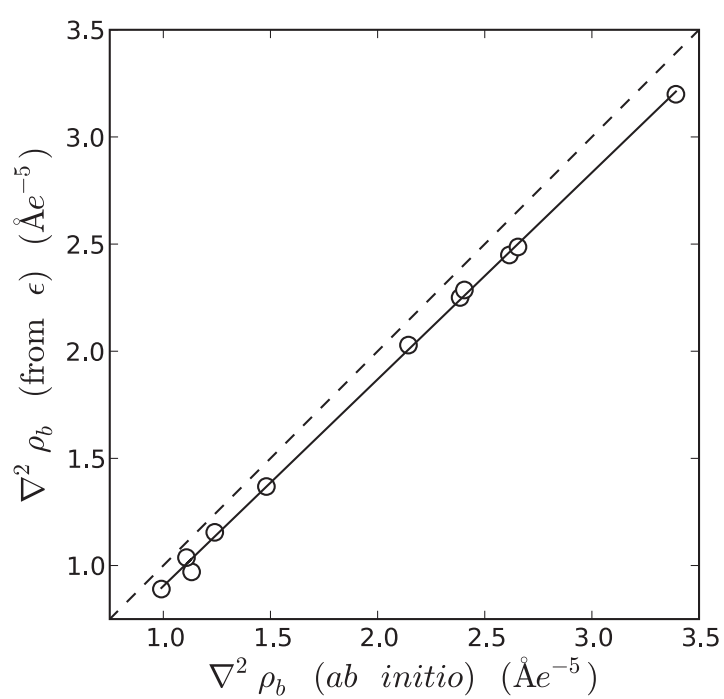

(a)

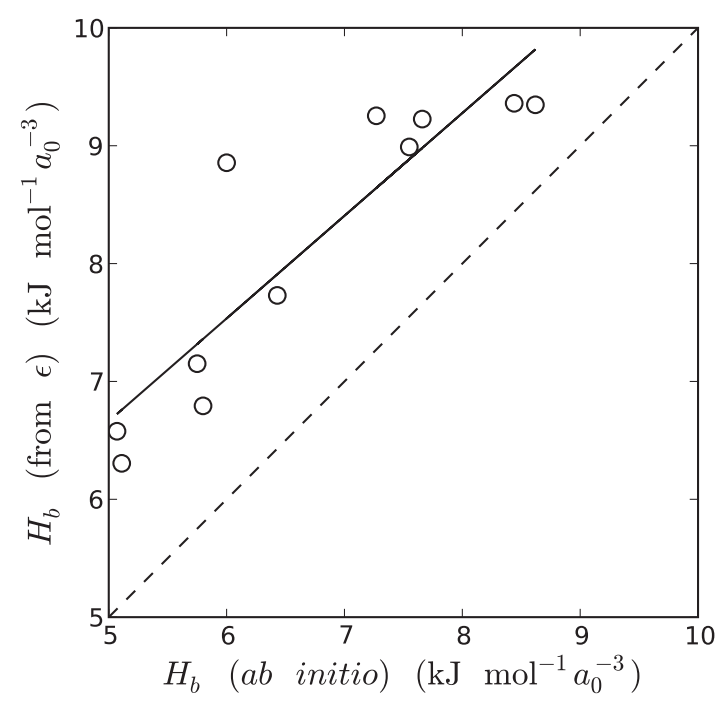

(b)

FIG. 12. (a) $\nabla^{2} \rho_{b}$ and (b) $H_{b}$ estimated from Table I and the effective $\epsilon$ for each FH $\cdots$ FY complex vs $a b$ initio values from Ref. 14. Solid lines are the linear fits in Table II. Dashed lines are guide to the eyes for the exact correspondence between magnitudes.

timated from $\epsilon$ are not equal to the ab initio values but proportional to them. It must be noticed that the linear dimer $(\mathrm{FH})_{2}$, which has been taken as a reference system for a set of FH $\cdots$ FY complexes at their fully optimized geometry, does not belong to this set of complexes. Indeed, $(\mathrm{FH})_{2}$ is

TABLE II. Linear fits $y_{\epsilon}=m y_{c}+n$, where subindices $\epsilon$ and $c$ refer to values estimated from Eq. (11) and Table I and from Ref. 14, respectively.

\begin{tabular}{lccc}
\hline \hline$y$ & $m$ & $n$ & $R$ \\
\hline$\rho_{b}\left(e \AA^{-3}\right)$ & $0.96(2)$ & $-0.016(2)$ & 0.9986 \\
$\lambda_{\|, b}\left(e \AA^{-5}\right)$ & $0.94(1)$ & $-0.12(2)$ & 0.9995 \\
$\lambda_{\perp, b}\left(e \AA^{-5}\right)$ & $0.92(1)$ & $0.15(1)$ & 0.9969 \\
$G_{b}\left(\mathrm{~kJ} \mathrm{~mol}^{-1} a_{0}^{-3}\right)$ & $0.88(1)$ & $1.9(6)$ & 0.9991 \\
$V_{b}\left(\mathrm{~kJ} \mathrm{~mol}^{-1} a_{0}^{-3}\right)$ & $0.91(2)$ & $6.9(7)$ & 0.9987 \\
$\nabla^{2} \rho_{b}\left(e \AA^{-5}\right)$ & $0.97(1)$ & $-0.06(2)$ & 0.9994 \\
$H_{b}\left(\mathrm{~kJ} \mathrm{~mol}^{-1} a_{0}^{-3}\right)$ & $0.87(15)$ & $2(1)$ & 0.8930 \\
\hline \hline
\end{tabular}


only included in its bent conformation. However, in spite of this, the linear dimer $(\mathrm{FH})_{2}$ is close enough to the set of complexes to provide reliable estimations for their $\mathrm{BCP}$ properties.

This very simple example shows that the effects of the perturbations induced by either a substituent $\mathrm{Y}$ or an external electric field $\epsilon$ are equivalent and, therefore, the effect of $\mathrm{Y}$ on the topology of $\rho(\mathbf{r})$ in the hydrogen bond can be nicely represented in terms of an effective electric field along the bond direction. The success in representing the substituent effect by an external electric field is explained because the complexes are very similar between them, as they present the same donor molecule. Up to which point a set of complexes can be grouped in a family equivalent to a reference system with an external electric field and which is the best choice for the reference system are questions that deserve further investigation, but they fall outside the scope of this paper.

\section{E. Dissociation energy}

Relationships between the dissociation energy $\left(D_{e}\right)$, defined as the energy difference between the complex at its equilibrium configuration and the isolated monomers, and the $\rho(\mathbf{r})$ properties at BCP have been several times pointed out. Among these relationships, the proportionality of $D_{e}$ with $\rho_{b}$ and with $\nabla^{2} \rho_{b}$ has been proposed, first observed in theoretical analyses of $\mathrm{N} \cdots \mathrm{HF}$ complexes ${ }^{4,5}$ and afterward extended to many other hydrogen bond types, ${ }^{46}$ including $\mathrm{CH} \cdots \pi$ interactions, ${ }^{47}$ and to stacking interactions. ${ }^{48}$ While in the case of $\rho_{b}$ the same linear relationship roughly spans from very weak interactions to covalent bonds, ${ }^{49}$ the relationship between $\nabla^{2} \rho_{b}$ and $D_{e}$ seems to be more complex and strongly deviates from a linear dependence for very strong and very weak interactions. ${ }^{50}$ Another linear relationship has been observed between $V_{b}$ and $D_{e}$ from the analysis of experimentally determined electron density distributions. ${ }^{7}$

If the perturbation that follows upon application of an external field on a single complex $\mathrm{X}-\mathrm{D}-\mathrm{H} \cdots \mathrm{A}-\mathrm{Y}$ is analogous to the effect of the exchange of substituents $(\mathrm{X}, \mathrm{Y})$ in the same family of complexes, those relationships should be here observed with this methodology. Thus, taking $\epsilon$ as a parameter representing the environment effect on the system, $D_{e}$ appears as the difference between the energy of the complex at the equilibrium configuration upon $\epsilon$ minus the energy of the isolated monomers, also at their equilibrium configuration upon the same $\epsilon$. In this way, $D_{e}(\epsilon)$ can be described in terms of the interaction energy as $-E_{i}\left(\epsilon, d_{\mathrm{eq}}(\epsilon)\right)$, where $d_{\mathrm{eq}}(\epsilon)$ is the equilibrium position with the field $\epsilon$ (Fig. 7).

The variation in the dissociation energy induced by the external electric field can be understood in terms of the polarization of the MOs that, in the case $\epsilon>0$, has a stabilizing effect on both short-range and long-range contributions to the interaction energy. The polarization of the MOs, along with the shift to shorter $d_{\mathrm{eq}}$ distances of the minimum of the interaction energy, affects the $\rho(\mathbf{r})$ properties, in particular at BCP. Thus, while the interaction remains as a closed shell, both the polarization and the shortening of the hydrogen bond distance increase the local electric field and the Pauli repulsion in the bonding region, with the concomitant increase in $\rho_{b}, \nabla^{2} \rho_{b},\left|V_{b}\right|, G_{b}, \lambda_{\|, b}$, and $\left|\lambda_{\perp, b}\right| .^{33}$ As the variations in the $\rho(\mathbf{r}) \mathrm{BCP}$ properties and the dissociation energy can be traced back to the same origin, namely, the polarization of the MOs of the donor and acceptor molecules, it is not surprising to find correlations between the $\rho(\mathbf{r})$ topological properties and $D_{e}$.

The values of $D_{e}$, calculated at the equilibrium geometries for each applied field, are represented against the corresponding values of $\rho_{b}, \nabla^{2} \rho_{b}, V_{b}, G_{b}, \lambda_{\|, b}$, and $\lambda_{\perp, b}$ in Fig. 13. Comparing the six figures, $G_{b}$ and $\lambda_{\|, b}$ present a clear linear dependence with $D_{e}$, while the other properties present a more complex dependence, even if they can be fairly fitted by a linear function. Figure 13 also shows the relationships expected from the fits shown in Table I and Eq. (7), setting $d_{\mathrm{FH}}=d_{\mathrm{eq}}\left[d_{\mathrm{eq}}=d_{\mathrm{eq}}(\epsilon)\right.$ from Eq. (9) $]$. Although these last relationships are in fair agreement with the minimized complexes, there are significant differences for the strongest interaction, which corresponds to the largest positive $\epsilon$. As stated before, the fitting functions obtained from reaction coordinates provide an overall good description of the topological properties and $D_{e}$ in the whole range of $d_{\mathrm{FH}}$ distances and external fields analyzed in this work, but they can be inaccurate in describing fine details of these properties at $d_{\mathrm{eq}}(\epsilon)$.

In the range of applicability of the dependencies at equilibrium geometries $\left[1.60(3)<d_{\mathrm{FH}}<2.33(4) \AA\right]$, much smaller than the range of $d_{\mathrm{FH}}$ distances explored for the reaction coordinate dependencies $\left(1.2<d_{\mathrm{FH}}<3.0 \AA\right)$, the interaction is of pure closed-shell type. ${ }^{14}$ For the latter, the covalent character of the interaction is not relevant, a result that agrees with the classification of hydrogen bonds from their interaction energies, ${ }^{3}$ stating that covalency starts to be significant for medium-strong hydrogen bonds $\left[D_{e} \geqslant 60 \mathrm{~kJ} \mathrm{~mol}^{-1}\right.$, which roughly corresponds to $d_{\mathrm{FH}}$ $\lesssim 1.60 \AA$, as estimated from $D_{e}=-E_{i}=3(2)+0.48(4) V_{b}$ and $V_{b}=-30350(900) \exp \left(-3.392(7) d_{\mathrm{FH}}\right)$ (Ref. 14)]. Although the interaction can be considered as of pure closed-shell type, the deviations from proportional correspondences in Fig. 13 are related to a starting $\rho(\mathbf{r})$ reorganization permitting the transition to a covalent interaction. Thus, as the field is more positive, the pressure on the electrons (represented by $V_{b}$ ) grows faster than the dissociation energy. This feature corresponds to a more important descreening felt by the hydrogen of the donor molecule and to the concomitant increase in the electrostatic field in the bonding region that favors the transition to a covalent bond.

The $\mathrm{H} \cdots \mathrm{A}$ distance has been also proposed as an indicator of the strength of the interaction, as it has been observed that this distance shortens with the increase in $D_{e}$, presenting an exponential dependence ${ }^{7}$ that is approximately linear if the range of interaction distances is small enough. $^{51,52}$ As previously shown for the linear $(\mathrm{FH})_{2}$, the polarization induced by the external electric field determines the variations in $D_{e}$ and $d_{\mathrm{eq}}$ through the dependence of the interaction energy on $\epsilon$ (Eq. (6)). At equilibrium geometries, 


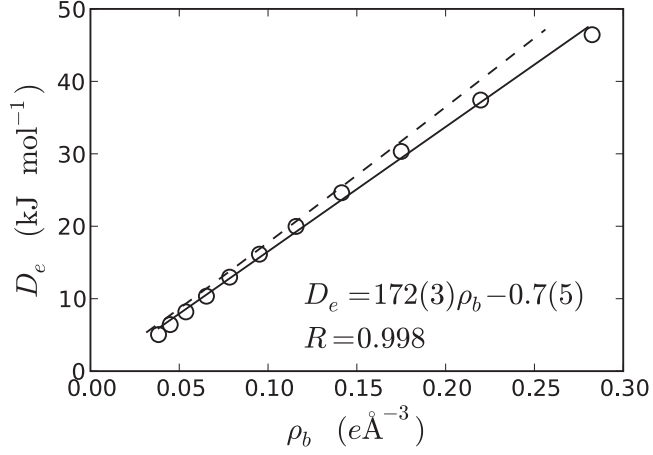

(a)

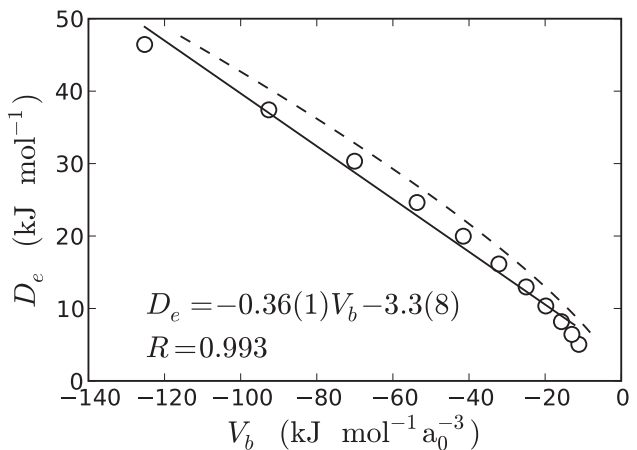

(c)

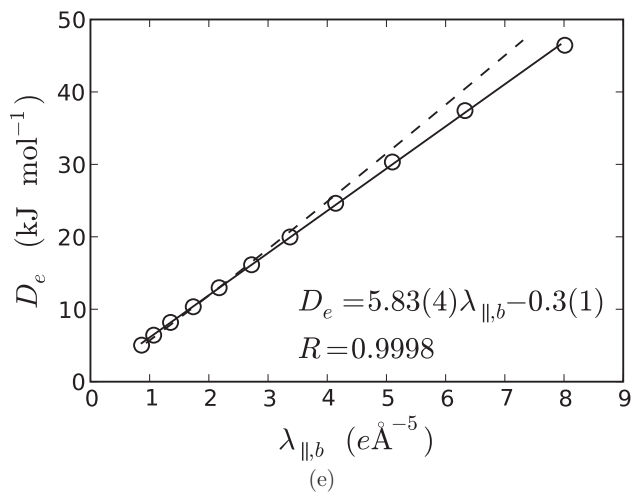

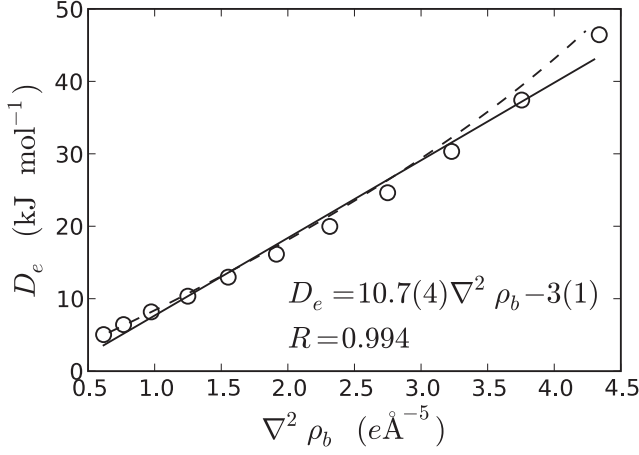

(b)

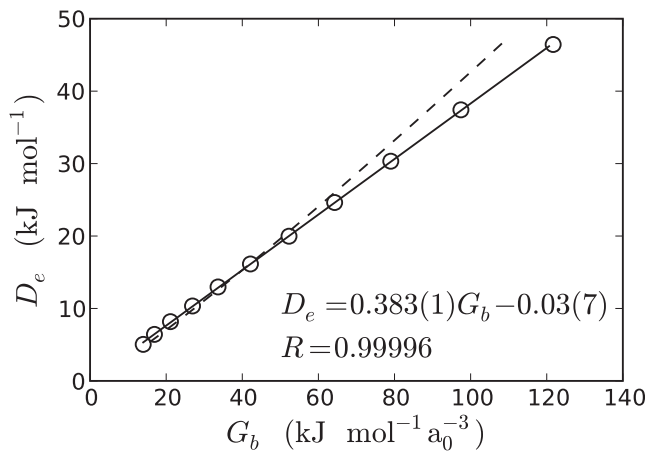

(d)

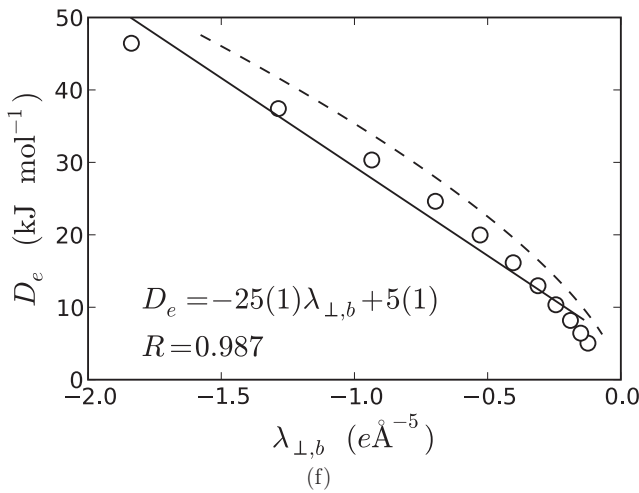

FIG. 13. $D_{e}$ vs (a) $\rho_{b}$, (b) $\nabla^{2} \rho_{b}$, (c) $V_{b}$, (d) $G_{b}$, (e) $\lambda_{\|, b}$, and (f) $\lambda_{\perp, b}$ at the equilibrium geometry obtained for each applied field. Solid lines correspond to the fit to a linear fitting function. Dashed lines correspond to the dependencies calculated from the fitting function shown in Table I and Eq. (7).

the dependence of $D_{e}$ on $d_{\mathrm{FH}}$ in the linear $(\mathrm{FH})_{2}$ can be obtained by substituting Eq. (11) in Eq. (7), and setting $d_{\mathrm{FH}}$ $=d_{\mathrm{eq}}(\epsilon)$, yielding

$$
D_{e}\left(d_{\mathrm{eq}}\right)=K e^{-\left(b_{1} k_{2}+b_{2} k_{1}\right) /\left(k_{1}+k_{2}\right) d d_{\mathrm{eq}}}
$$

with

$$
K=\left(a_{1}^{k_{2}} a_{2}^{k_{1}}\right)^{1 /\left(k_{1}+k_{2}\right)}\left(\left(\frac{b_{1}}{b_{2}}\right)^{k_{2} /\left(k_{1}+k_{2}\right)}-\left(\frac{b_{2}}{b_{1}}\right)^{k_{1} /\left(k_{1}+k_{2}\right)}\right) .
$$

The exponential dependence of $D_{e}$ on $d_{\mathrm{eq}}$ appears as a direct consequence of the $E_{i}\left(d_{\mathrm{FH}}, \epsilon\right)$ exponential dependence with both $\epsilon$ and $d_{\mathrm{FH}}$. The decay of $D_{e}$ with $d_{\mathrm{eq}}$ depends on the decay with $d_{\mathrm{FH}}$ of both terms of $E_{i}$, represented by $b_{1}$ and $b_{2}$, and on the response of the interaction energy to $\epsilon$, represented by $k_{1}$ and $k_{2}$.
The exponent in Eq. (12) can be rewritten as

$$
\frac{\frac{b_{1}}{k_{1}}+\frac{b_{2}}{k_{2}}}{\frac{1}{k_{1}}+\frac{1}{k_{2}}},
$$

showing the addition of terms related to each contribution to the interaction energy in Eq. (6). As $b_{1} / k_{1} \approx 9 b_{2} / k_{2}$ [Eq. (7)], the contribution of the long-range term is more important in the decay of $D_{e}$ with $d_{\text {eq }}$. Although the effect of the MO reorganization cannot be ignored, it is the mutual polarization that controls the dependence of $D_{e}$ with $d_{\mathrm{eq}}$. This dependence is represented along with Eq. 12 in Fig. 14(a). The log-linear plot clearly shows the exponential dependence, which is also nicely reproduced by Eq. (12) in the range of equilibrium distances considered. This result points the equilibrium distance as an excellent indicator of the strength of 


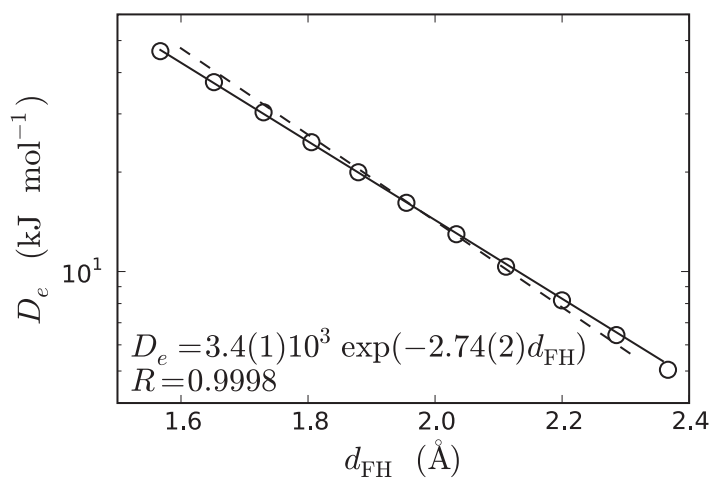

(a)

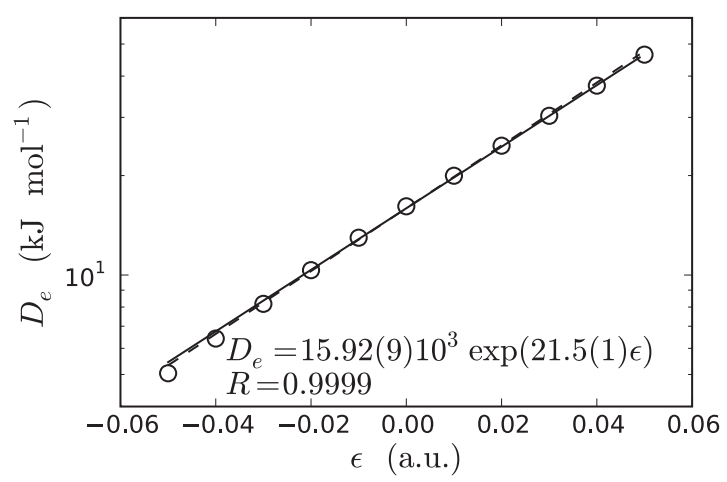

(b)

FIG. 14. Log-linear plots of $D_{e}$ vs (a) $d_{\mathrm{FH}}$ and (b) $\epsilon$ at the equilibrium distance obtained for each applied field (Eq. (9)). Solid lines correspond to the exponential fittings given in the graphs, while dashed lines are calculated from Eqs. (7), (9), and (12).

the interaction, like $G_{b}$ or $\lambda_{\|, b}$, besides the information that the former gives on the form of the interaction energy surface.

According to the interpretation of $\epsilon$ in terms of a parameter that can be associated with an external perturbation on a given interaction, the molecular environment tunes, through $\epsilon$, the bonding distance [Eqs. (9) and (11)] and the dissociation energy (Fig. 14(b)). Recently, the influence of the crystalline environment on molecular dipoles has been successfully represented in terms of an electric field. ${ }^{16}$ In molecular crystals, it is expected that the perturbation induced by the crystalline environment on a hydrogen bonding interaction could be represented by an effective parameter $\epsilon$, fixing the topological properties at BCP to the magnitudes experimentally observed for the dependencies with the bonding distance. ${ }^{6}$ The use of this effective electric field could thus permit the modeling of the $\mathrm{H} \cdots \mathrm{A}$ interaction potential in the crystal phase by tuning (i) the equilibrium distance, (ii) the potential-well depth (i.e., the interaction energy), and (iii) the curvature at the minimum (i.e., the force constant at $d_{\mathrm{eq}}$ ) through $\epsilon$. However, it should be noted that while the hydrogen bonded system tries to accommodate to this equilibrium configuration, steric constraints imposed by the crystalline environment could avoid its full relaxation. Altogether, the scattering of values around the empirical dependencies that is observed for the $\mathrm{BCP}$ properties of an $\mathrm{X}-\mathrm{D}-\mathrm{H} \cdots \mathrm{A}-\mathrm{Y}$ family ${ }^{6}$ can be due, besides the experimental noise, to the different $\epsilon$ magnitudes that each particular crystalline surrounding exerts on its corresponding family member and to a nonrelaxed conformation resulting from steric constraints.

\section{CONCLUSIONS}

In the case of the linear $(\mathrm{FH})_{2}$ complex, the effect of a parallel external electric field on the topological properties at the hydrogen bond BCP can be explained in terms of a larger polarizability of the bonding orbital of the donor molecule with respect to that of the acceptor one. Thus, a field pointing from the donor to the acceptor polarizes this orbital away from the bonding region, relaxing the Pauli repulsion and favoring the transition of the hydrogen bond to a covalent bond. On the other hand, an external field pointing from the acceptor to the donor pushes the bonding orbital of the donor toward the acceptor lone pairs and weakens the hydrogen bond, increasing the Pauli repulsion and therefore hindering the formation of the covalent bond by favoring the closedshell character of the interaction.

The dependence of the interaction energy on the $\mathrm{F} \cdot \mathrm{H}$ distance can be explained from the integrated properties. For long enough distances, the complex is stabilized as the molecules approach, due to an increase in the mutual polarization that strengthens the interaction. At short distances, the energy of the complex is controlled by the destabilization of the terminal atoms, as a result of the weakening of the covalent bonds in both molecules. An external electric field from the donor to the acceptor increases the mutual polarization and stabilizes the complex, shortening the equilibrium distance. In addition, a field from the acceptor to the donor decreases the mutual polarization, destabilizing the complex and lengthening the equilibrium distance.

The application of external electric fields appears as an interesting method for the evaluation of equilibrium distances, dissociation energies, and $\rho(\mathbf{r})$ BCP properties in families of complexes $\mathrm{X}-\mathrm{D}-\mathrm{H} \cdots \mathrm{A}-\mathrm{Y}$. The external field induces a perturbation on the MOs that shows in the dependencies of the BCP properties and the interaction energy on the $\mathrm{H} \cdots \mathrm{A}$ distance. As a result of this perturbation, the position of the minimum of the interaction energy, which corresponds to the equilibrium distance, is displaced. By taking the values at the equilibrium position as a function of the applied field, it is possible to study the continuous variation of the BCP properties while keeping in all cases the system at its equilibrium configuration. These dependencies are analogous to those observed in families of complexes, with the difference that the applied field avoids the scattering of values that, due to the variation of the $\mathrm{X}$ and $\mathrm{Y}$ molecular groups, is found for the dependence of each property around the overall curve associated with the $\mathrm{D}-\mathrm{H} \cdots \mathrm{A}$ interaction.

The external electric field can be considered as a parameter associated to the effect of the atomic and molecular environment on the considered interaction. The value of the external field fixes the interaction energy, the equilibrium distance and the BCP properties at this geometry, all of them appearing intrinsically related to each other.

In the particular case of the linear dimer $(\mathrm{FH})_{2}$, a linear relationship is observed between the applied field $\epsilon$ and the 
equilibrium distance $d_{\mathrm{eq}}(\epsilon)$. In addition, based on the modeling of $E_{i}$ in terms of the sum of two exponential contributions, it is demonstrated that the dissociation energy, defined as the interaction energy at the equilibrium configuration, decays exponentially with the equilibrium distance obtained for each $\epsilon$. While most BCP properties present roughly linear dependencies on the dissociation energy, both the kinetic energy density $G_{b}$ and the curvature of the electron density along the bonding direction $\lambda_{\|, b}$ present an almost exact proportionality with the dissociation energy. As these quantities can be directly determined $\left(\lambda_{\|, b}\right)$ or indirectly estimated $\left(G_{b}\right)$ from high resolution $\mathrm{x}$-ray diffraction analysis, this result suggests that they can be used as very good indicators of the strength of the hydrogen bond in the analysis of experimental electron distributions. This is particularly underlined for $\lambda_{\|, b}$, which was already pointed out as the best experimentally determined topological parameter. ${ }^{6}$ However, it must be noticed that those dependencies have been here observed at equilibrium configuration, while in crystalline environments steric constraints can avoid the full relaxation of the interaction to its equilibrium geometry. Thus, for the analysis of experimental electron densities, a good understanding of the relationship between the interaction energy and the BCP topological and energetic properties is needed not only at equilibrium distances but also out of equilibrium configurations.

Finally, the use of the methodology developed in this work appears as an important issue toward the knowledge of electric fields generated by atomic environments in gas, liquid, and crystalline phases, and it will be treated in forthcoming articles.

\section{ACKNOWLEDGMENTS}

This work was supported by the Spanish Ministerio de Educación y Ciencia (MEC) (Grant Nos. MAT2006-13572C02-01 and CTQ2007-61901/BQU) and Generalitat de Catalunya (Grant No. 2005SGR-452). I.M. also thanks the MEC for a Juan de la Cierva fellowship. Thanks are due to CTI (CSIC) for generous allocation of computational resources. E.E. thanks Professor M. A. Spackman for helpful comments on the magnitudes of crystal electric fields.

${ }^{1}$ R. F. W. Bader, Atoms in Molecules: A Quantum Theory (Clarendon, Oxford, 1990)

${ }^{2}$ The Quantum Theory of Atoms in Molecules, edited by C. F. Matta and R. J. Russell (Wiley-VCH, New York, 2007).

${ }^{3}$ G. A. Jeffrey, An Introduction to Hydrogen Bonding (Oxford University Press, New York, 1997).

${ }^{4}$ R. J. Boyd and S. C. Choi, Chem. Phys. Lett. 120, 80 (1985)

${ }^{5}$ R. J. Boyd and S. C. Choi, Chem. Phys. Lett. 129, 62 (1986).

${ }^{6}$ E. Espinosa, M. Souhassou, H. Lachekar, and C. Lecomte, Acta Crystallogr., Sect. B: Struct. Sci. 55, 563 (1999).

${ }^{7}$ E. Espinosa, E. Molins, and C. Lecomte, Chem. Phys. Lett. 285, 170 (1998).

${ }^{8}$ E. Espinosa and E. Molins, J. Chem. Phys. 113, 5686 (2000)

${ }^{9}$ M. J. T. Jordan and J. E. Del Bene, J. Am. Chem. Soc. 122, 2101 (2000).

${ }^{10}$ J. Bevitt, K. Chapman, D. Crittenden, M. J. T. Jordan, and J. E. Del Bene, J. Phys. Chem. A 105, 3371 (2001).

${ }^{11}$ J. E. Del Bene and M. J. T. Jordan, J. Phys. Chem. A 106, 5385 (2002).

${ }^{12}$ M. Ramos, I. Alkorta, J. Elguero, N. Golubev, G. Denisov, H. Benedict, and H.-H. Limbach, J. Phys. Chem. A 101, 9791 (1997).
${ }^{13}$ I. Rozas, I. Alkorta, and J. Elguero, Chem. Phys. Lett. 275, 423 (1997).

${ }^{14}$ E. Espinosa, I. Alkorta, J. Elguero, and E. Molins, J. Chem. Phys. 117, 5529 (2002)

${ }^{15}$ E. Espinosa, I. Alkorta, I. Mata, and E. Molins, J. Phys. Chem. A 109, 6532 (2005)

${ }^{16}$ M. A. Spackman, P. Munshi, and D. Jayatilaka, Chem. Phys. Lett. 443, 87 (2007).

${ }^{17}$ R. Kahn, E. C. D. Lara, and K. D. Moller, J. Chem. Phys. 83, 2653 (1985).

${ }^{18}$ H. Lehle, J. M. Kriegl, K. Nienhaus, P. Deng, S. Fengler, and U. Nienhaus, Biophys. J. 88, 1978 (2005).

${ }^{19}$ I. T. Suydam, C. D. Snow, V. S. Pande, and S. G. Boxer, Science 313, 200 (2006).

${ }^{20}$ O. Galvez, P. C. Gomez, and L. F. Pacios, J. Chem. Phys. 115, 11166 (2001).

${ }^{21}$ M. J. Frisch, G. W. Trucks, H. B. Schlegel et al., GAussian 03, Revision E.01, Gaussian, Inc., Wallingford, CT, 2004.

${ }^{22}$ C. Møller and M. S. Plesset, Phys. Rev. 46, 618 (1934).

${ }^{23}$ M. J. Frisch, J. A. Pople, and J. S. Binkley, J. Chem. Phys. 80, 3265 (1984).

${ }^{24}$ F. Biegler-König, R. Bader, and T.-H. Tang, J. Comput. Chem. 3, 317 (1982).

${ }^{25}$ P. L. A. Popelier, MORPHY98 0.2, a topological analysis program, UMIST, Manchester, England, 1999.

${ }^{26}$ I. Alkorta and O. Picazo, Arkivoc ix, 305 (2005).

${ }^{27}$ R. F. W. Bader, J. Phys. Chem. A 102, 7314 (1998)

${ }^{28}$ Y. A. Abramov, Acta Crystallogr., Sect. A: Found. Crystallogr. 53, 264 (1997).

${ }^{29}$ E. Espinosa, C. Lecomte, and E. Molins, Chem. Phys. Lett. 300, 745 (1999).

${ }^{30}$ E. Espinosa, I. Alkorta, I. Rozas, J. Elguero, and E. Molins, Chem. Phys. Lett. 336, 457 (2001).

${ }^{31}$ A. E. Reed, L. A. Curtiss, and F. Weinhold, Chem. Rev. (Washington, D.C.) 88, 899 (1988).

${ }^{32}$ I. Rozas, I. Alkorta, and J. Elguero, J. Am. Chem. Soc. 122, 11154 (2000).

${ }^{33}$ I. Mata, E. Molins, I. Alkorta, and E. Espinosa, J. Phys. Chem. A 111, 6425 (2007).

${ }^{34}$ U. Koch and P. L. A. Popelier, J. Phys. Chem. 99, 9747 (1995).

${ }^{35}$ P. M. Dominiak, A. Makal, P. R. Mallinson, K. Trzcinska, J. Eilmes, E. Grech, M. Chruszcz, W. Minor, and K. Woźniak, Chem.-Eur. J. 12, 1941 (2006).

${ }^{36}$ E. R. Davidson, Int. J. Quantum Chem. 98, 317 (2004).

${ }^{37}$ M. Springborg, Phys. Rev. Lett. 59, 2287 (1987).

${ }^{38}$ A. Masunov, J. Dannenberg, and R. Contreras, J. Phys. Chem. A 105, 4737 (2001).

${ }^{39}$ H. Umeyama and K. Morokuma, J. Am. Chem. Soc. 99, 1316 (1977).

${ }^{40}$ R. Gora, S. Grabowski, and J. Leszczynski, J. Phys. Chem. A 109, 6397 (2005).

${ }^{41}$ A. E. Reed, F. Weinhold, L. A. Curtiss, and D. J. Pochatko, J. Chem. Phys. 84, 5687 (1986).

${ }^{42}$ I. Alkorta, I. Rozas, and J. Elguero, Theor. Chem. Acc. 99, 116 (1998).

${ }^{43}$ J. E. Del Bene, W. B. Person, and K. Szczepaniak, Chem. Phys. Lett. 247, 89 (1995).

${ }^{44}$ E. Lee, B. Hong, J. Lee, J. Kim, D. Kim, Y. Kim, P. Tarakeshwar, and K. Kim, J. Am. Chem. Soc. 127, 4530 (2005).

${ }^{45}$ M. J. T. Jordan and K. C. Thompson, Chem. Phys. Lett. 370, 14 (2003).

${ }^{46}$ S. Grabowski, J. Phys. Chem. A 105, 10739 (2001).

${ }^{47}$ E. Cubero, M. Orozco, P. Hobza, and F. Luque, J. Phys. Chem. A 103, 6394 (1999)

${ }^{48}$ R. Parthasarathi and V. Subramanian, Struct. Chem. 16, 243 (2005).

${ }^{49}$ R. Parthasarathi, V. Subramanian, and N. Sathyamurthy, J. Phys. Chem. A 110, 3349 (2006).

${ }^{50}$ S. Wojtulewski and S. J. Grabowski, J. Mol. Struct. 605, 235 (2002).

${ }^{51}$ S. Scheiner, S. Grabowski, and T. Kar, J. Phys. Chem. A 105, 10607 (2001).

${ }^{52}$ I. Alkorta and J. Elguero, J. Am. Chem. Soc. 124, 1488 (2002).

${ }^{53}$ See EPAPS Document No. E-JCPSA6-130-014904 for the numerical data for the surfaces of the interaction energy and the critical point properties. For more information on EPAPS, see http://www.aip.org/pubservs/ epaps.html. 